\title{
SkyMapper stellar parameters for Galactic Archaeology on a grand-scale
}

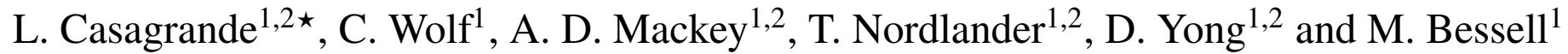 \\ ${ }^{1}$ Research School of Astronomy and Astrophysics, Mount Stromlo Observatory, The Australian National University, ACT 2611, Australia \\ ${ }^{2}$ ARC Centre of Excellence for All Sky Astrophysics in 3 Dimensions (ASTRO 3D)
}

Received; accepted

\begin{abstract}
The SkyMapper photometric surveys provides uvgriz photometry for several millions sources in the Southern sky. We use DR1.1 to explore the quality of its photometry, and develop a formalism to homogenise zero-points across the sky using stellar effective temperatures. Physical flux transformations, and zero-points appropriate for this release are derived, along with relations linking colour indices to stellar parameters. Reddening-free pseudo-colours and pseudo-magnitudes are also introduced. For late-type stars which are best suited for Galactic Archaeology, we show that SkyMapper+2MASS are able to deliver a precision better than $100 \mathrm{~K}$ in effective temperatures (depending on the filters), $\sim 0.2$ dex for metallicities above -2 , and a reliable distinction between M-dwarfs and -giants. Together with astrometric and asteroseismic space mission, SkyMapper promises to be a treasure trove for stellar and Galactic studies.
\end{abstract}

Key words: stars: fundamental parameters - stars: late-type - Galaxy: stellar content

\section{INTRODUCTION}

Photometric systems and filters are designed to be sensitive to certain spectral features. In the case of stars, these features are driven by physical parameters such as effective temperature, gravity and metallicity. To accomplish this goal, filter systems are tailored to select regions in stellar spectra where the variations of the atmospheric parameters leave their characteristic traces with enough prominence to be detected. A large number of photometric systems exists nowadays for different scientific purposes (e.g., Bessell 2005 for a review), and indeed the advent of large scale photometric surveys is impacting every area of astrophysics (e.g., Ivezić et al.2012 for a review).

Among the many photometric surveys is SkyMapper ${ }^{1}$, a $1.35 \mathrm{~m}, 32 \mathrm{CCDs}$, automated wide-field survey telescope located at Siding Spring Observatory (Australia), undertaking a multi-epoch photometric survey of the entire Southern sky (Keller et al. 2007, Wolf et al. 2018). The SkyMapper photometric system builds on the success of the griz filters used by the Sloan Digital Sky Survey (Fukugita et al. 1996, Doi et al. 2010), with the added value of the $u v$ bands, designed to be strongly sensitive to stellar parameters. The SkyMapper $u$ band mimics the Strömgren $u$ filter, which covers the Balmer discontinuity and provides good temperature sensitivity in hot stars, and gravity sensitivity across A, F and G spectral types (e.g., Strömgren 1951; Árnadóttir et al. 2010). The SkyMapper $v$ filter is instead different from the Strömgren $v$, and

\footnotetext{
^ Email:luca.casagrande@anu.edu.au
}

1 http://skymapper.anu.edu.au/ shifted $\sim 200 \AA$ towards the blue to be even more sensitive at low metallicities, similarly to the DDO38 filter (McClure 1976). The only other existing all-sky survey measuring intermediate $u v$ photometry is the Geneva-Copenhagen Survey (GCS, Nordström et al. 2004) but at significantly brighter magnitudes than those probed by SkyMapper, and only for FG spectral types. Nevertheless, the GCS has clearly shown the power of intermediate Strömgren $u v$ photometry for Galactic studies. Indeed, early SkyMapper data has already been very successful at finding some of the most iron-poor stars in the Galaxy (e.g., Keller et al. 2014, Howes et al. 2016). A full description of the SkyMapper photometric system can be found in Bessell et al. (2011).

In 2017, SkyMapper made available ${ }^{2}$ its Data Release 1.1 (DR1.1) (Wolf et al. 2018), which provides uvgriz magnitudes for over 285 million objects across most of the southern sky $\left(17,200 \mathrm{deg}^{2}\right)$. Although the goal of SkyMapper is to deliver magnitudes in the $\mathrm{AB}$ system, when implementing a photometric system at the telescope it is not necessarily straightforward to adhere to the definition, and small zero-points offsets might be present. Knowledge of these offsets is important to assess the quality of the observations, to convert magnitudes into fluxes, as well as e.g., to compute theoretical synthetic colours to compare with observations (e.g., Casagrande \& VandenBerg 2018a b). Indeed, the first goal of

\footnotetext{
2 As explained in Wolf et al. 2018, the major improvement with respect to DR1 is a significant enhancement of the homogeneity of the photometric calibration. By default all queries in SkyMapper now return DR1.1 photometry, which is the one used in this paper.
} 
this paper is to assess the DR1.1 photometric standardization. In this process, we develop a new method to infer photometric zeropoints across the sky, and we provide corrections to place uvgriz photometry onto the $\mathrm{AB}$ system.

Over the next few years SkyMapper will deliver a uniquely powerful dataset to investigate stellar populations across the Galaxy, enabling studies in most areas of Galactic Archaeology. Thus, the second goal of this paper is to derive empirical calibrations relating basic stellar parameters $\left(T_{\text {eff }}, \log g\right.$ and $[\mathrm{Fe} / \mathrm{H}])$ to SkyMapper photometry. Stellar effective temperatures are derived implementing SkyMapper photometry into the InfraRed Flux Method (IRFM, Casagrande et al. 2010). The sensitivity of SkyMapper photometry to $[\mathrm{Fe} / \mathrm{H}]$ and $\log g$ is explored using over a quarter of a million stars in common between SkyMapper and the spectroscopic GALactic Archaeology with Hermes survey (GALAH, Buder et al.2018). We are able to compare photometric $T_{\text {eff }}$ from SkyMapper to spectroscopic ones from GALAH, as well as to explore the sensitivity of SkyMapper filters to stellar parameters. This exercise goes beyond the importance of cross-validating the two surveys. In fact, SkyMapper is ultimately expected to be magnitude-complete down to $g \simeq 22$, thus reaching several magnitudes fainter than GALAH, and approximately the same magnitude limit as Gaia, greatly enlarging the volume within which we can do Galactic Archaeology. The complementarity of SkyMapper to Gaia is enormous, especially at this stage when $B P$ and $R P$ spectra have not been released yet, meaning that Gaia stellar parameters are based only on $G_{\mathrm{BP}}, G$ and $G_{\mathrm{RP}}$ photometry, and thus subject to strong assumptions and degeneracies.

\section{THE SKYMAPPER SYSTEM}

A source having flux $f_{\lambda}$ and observed through a system response function $T_{\zeta}$ (which includes the total throughput reaching the observer over the bandpass $\zeta$ ) will have an $\mathrm{AB}$ magnitude (see e.g., Bessell \& Murphy 2012, Casagrande \& VandenBerg 2014, for the photon-counting formalism adopted here):

$$
m_{\zeta, \mathrm{AB}}=-2.5 \log \frac{\int_{v_{i}}^{v_{f}} f_{v} T_{\zeta} \mathrm{d} \ln v}{f_{v}^{0} \int_{v_{i}}^{v_{f}} T_{\zeta} \mathrm{d} \ln v}=-2.5 \log \frac{\int_{\lambda_{i}}^{\lambda_{f}} \lambda f_{\lambda} T_{\zeta} \mathrm{d} \lambda}{f_{v}^{0} c \int_{\lambda_{i}}^{\lambda_{f}} \frac{T_{\zeta}}{\lambda} \mathrm{d} \lambda},
$$

where $f_{v}^{0}=3.631 \times 10^{-20} \mathrm{erg} \mathrm{s}^{-1} \mathrm{~cm}^{-2} \mathrm{~Hz}^{-1}$ and $c$ is the speed of light. The actual realization of a photometric system at the telescope is far from the trivial definition given above. More often than not, zero-point corrections $\epsilon_{\zeta}$ are needed in each band to adhere to the definition (e.g., Eisenstein et al. 2006, Holberg \& Bergeron 2006 for the SDSS system). Thus, it is worth checking whether this is also the case for SkyMapper. Currently, each SkyMapper exposure is standardized as closely as possible to the AB system through comparison with APASS and 2MASS photometry (Henden et al. 2016, Skrutskie et al. 2006). SkyMapper standardized magnitudes (SM) can thus be written:

$$
m_{\zeta, \mathrm{SM}}=m_{\zeta, \mathrm{AB}}+\epsilon_{\zeta},
$$

where $\epsilon_{\zeta}$ allows for possible departure from the $\mathrm{AB}$ definition. In the most general form, these departures could depend on various factors such as position across the sky, magnitudes or colours. These effects are explored and discussed later in the paper.

Here, for each SkyMapper filter $(\zeta=u, v, g, r, i, z)$ we adopt the system response functions reported in Bessell et al. (2011). Since the SkyMapper system response functions are well characterized, one way of determining $\epsilon_{\zeta}$ is to use measured absolute spectrophotometry (i.e. $f_{\lambda}$ ) to compute $m_{\zeta, \mathrm{AB}}$ via Eq. (1). Comparison with observed SkyMapper magnitudes allows then to determine $\epsilon_{\zeta}$ via Eq. 22. The HST CALSPEC ${ }^{3}$ library offers the most accurate absolute spectrophotometry available to date, which is of order of a few percent, or better for stars with STIS/NICMOS observations (Bohlin et al. 2001, Bohlin 2007, 2014). We remark that a systematic uncertainty of order 1 percent in absolute flux translates into $2.5 \log (1.01) \simeq 0.01 \mathrm{mag}$ zero-point uncertainty. We compute photometric errors by taking into account systematic and statistical errors as reported for each absolute flux in CALSPEC. For STIS/NICMOS observations the impact of statistical errors is usually smaller, as they mostly compensate over a bandwidth. We find 11 stars in SkyMapper that also have CALSPEC absolute spectrophotometry, and Figure 1 shows the difference between the magnitudes observed and those computed via Eq. (1).

From Figure 1, $u$ is the only band displaying a $>3 \sigma$ offset from the $\mathrm{AB}$ system. $v$ also seems to be offset, but with a large scatter, the weighted difference and weighted sample variance not changing significantly if we were exclude the biggest outlier $(0.030 \pm 0.034$ instead of $0.027 \pm 0.043) . g$ and $z$ are consistent with being on the $\mathrm{AB}$ system, whereas small offsets are present for the $r$ and $i$ band, but those are only marginally significant (around 1 and $2 \sigma$, respectively). The minimal offset and typical 0.02 mag scatters for the griz filters support the conclusion of Wolf et al. (2018), who found a scatter of 2 percent with respect to the AB photometry from Pan-STARRS1. In Figure 1 the error bars of most points reach the zero-point corrected dashed-lines, except for $v$ band. This band is also characterized by a rather large scatter, which warrants further investigation. As we discuss in the next Section, a larger number of spectrophotometric standards across the sky would be necessary to draw a firmer conclusion. With this goal in mind, in the next Section we develop a new method to derive robust photometric zero-points using a cohort of stars across the sky.

\section{PHOTOMETRIC ZERO-POINTS FROM THE ABSOLUTE $T_{\text {eff }}$ SCALE}

In this section we explore an alternative approach to derive photometric zero-points for the SkyMapper system. To do so, we use the IRFM, which provides a nearly model independent and elegant technique for determining stellar effective temperatures (e.g., Blackwell et al. 1979, 1980). The IRFM relies on the ratio between the bolometric $\left(\mathcal{F}_{\text {bol }}\right)$ and the infrared monochromatic flux $\left(\mathcal{F}_{\text {IR }}\right)$ of a star measured on the Earth. Both quantities are determined observationally. This ratio is compared to the one defined on a stellar surface element, i.e. the bolometric flux $\sigma T_{\text {eff }}^{4}$ and the theoretical surface infrared monochromatic flux:

$$
\frac{\mathcal{F}_{\text {bol }}(\text { Earth })}{\mathcal{F}_{\text {IR }}(\text { Earth })}=\frac{\sigma T_{\text {eff }}^{4}}{\mathcal{F}_{\text {IR }}(\text { model })} .
$$

When working in the Rayleigh-Jeans tail, the model infrared flux is largely dominated by the continuum and relatively easy to compute, with a roughly linear dependence on $T_{\text {eff }}$ and very little affected by other stellar parameters, such as metallicity and surface gravity (as extensively tested in the literature, e.g, Blackwell et al. 1991, Alonso et al. 1996, Casagrande et al. 2006). The problem is therefore reduced to a proper derivation of stellar fluxes, and once this is done Eq. (3) can be rearranged to return its only unknown: $T_{\text {eff. }}$ The implementation we adopt for the IRFM uses multi-band optical and infrared photometry to recover $\mathcal{F}_{\text {bol }}$ and $\mathcal{F}_{\text {IR }}$. An iterative procedure

\footnotetext{
3 http://www.stsci.edu/hst/observatory/crds/calspec.html
} 

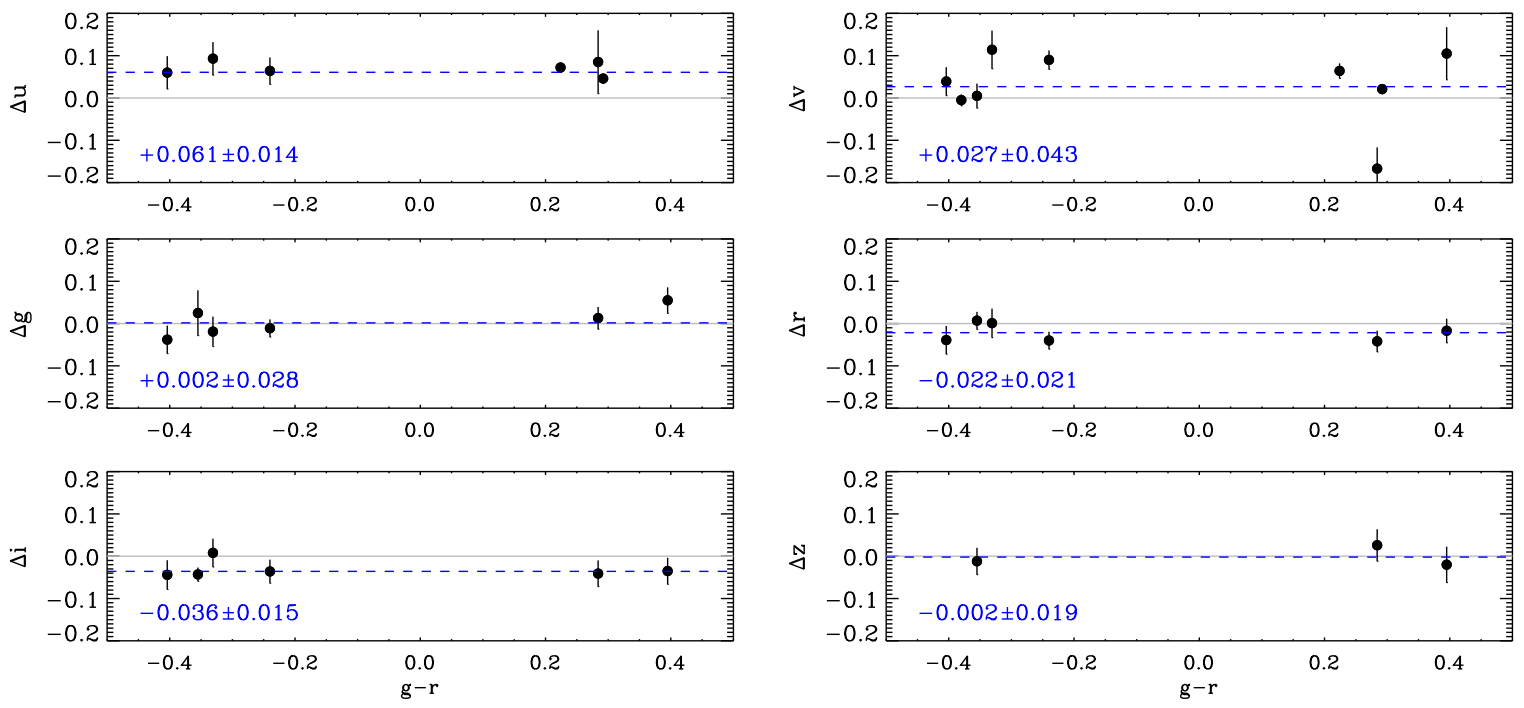

Figure 1. Observed SkyMapper minus AB magnitudes computed for stars in the CALSPEC library as function of their $g-r$ colour. For each band, only stars with no SkyMapper flags and no source within 15 arcsec have been retained. $\epsilon_{\zeta}$ are shown by dashed lines, with the weighted difference \pm the square root of the weighted sample variance indicated at the bottom of each panel.

in $T_{\text {eff }}$ is adopted to cope with the mild dependence on stellar parameters of the flux outside photometric bands (i.e., the bolometric correction), and of the theoretical surface infrared monochromatic flux. More specifically, for each star, we interpolate over a grid of synthetic model fluxes, starting with an initial estimate of the stellar effective temperature, and working at fixed $[\mathrm{Fe} / \mathrm{H}]$ and $\log g$ until convergence is reached in $T_{\text {eff }}$. Further details can be found in Casagrande et al. (2006, 2010). In essence, the method relies on a proper derivation of physical fluxes $\left(\mathrm{erg} \mathrm{s}^{-1} \mathrm{~cm}^{-2} \AA^{-1}\right)$ from magnitudes, meaning that the IRFM strongly depends on the absolute calibration underlying the photometric systems used into it. Without exaggeration, this is the most critical point when implementing the method (e.g., Blackwell et al. 1990). Casagrande et al. (2010) further highlighted how differences among IRFM scales in the literature can be simply explained by changing the absolute calibration of the adopted photometric systems, or equivalently using different photometric zero-points. This means that if we have a set of stars for which we accurately know their effective temperatures, we can implement a given photometric system (SkyMapper in this case) into the IRFM, and modify the adopted photometric zero-points until we are able to reproduce known effective temperatures.

As we have already discussed, the adopted implementation of the IRFM relies on multiband optical and infrared photometry to recover the bolometric flux. The infrared monochromatic flux is derived using only infrared magnitudes (2MASS $J H K_{s}$ in this case). The infrared absolute calibration and zero-points have already been determined in Casagrande et al. (2010) via solar-twins, and are kept unchanged here. An in-depth discussion of the flux associated to each SkyMapper magnitude is provided in the Appendix. For the sake of applying the IRFM, here it suffices to say that for each star we always require having 2MASS $J H K_{s}$ magnitudes (with combined photometric errors $<0.15 \mathrm{mag}$ ), plus at least one SkyMapper band. A band is used only if it has no SkyMapper flag, and no source within 15 arcsec. We also apply a threshold on photometric errors, $<0.1$ magnitude for $u$ and $v$ band, and $<0.04$ for griz, as we discuss in Section 3.3 .

To summarise, in our method for each star we input measured
Table 1. Average photometric zero-points $\epsilon_{\zeta}$ and characteristic parameters of the SkyMapper system.

\begin{tabular}{cccccc}
\hline & $\epsilon_{\zeta}$ & $\begin{array}{c}G(\lambda) \\
{\left[\mathrm{cm}^{-1} \AA^{-1}\right]}\end{array}$ & $\begin{array}{c}H(\lambda) \\
{\left[\mathrm{cm}^{-1}\right]}\end{array}$ & $\begin{array}{c}B w(\lambda) \\
{[\AA]}\end{array}$ & $\begin{array}{c}\lambda_{\text {eff }} \\
{[\AA]}\end{array}$ \\
\hline$u$ & $+0.032 \pm 0.020$ & 8.086 & 3446.6 & 426.2 & 3537 \\
$v$ & $+0.033 \pm 0.022$ & 6.796 & 2168.4 & 319.1 & 3874 \\
$g$ & $+0.009 \pm 0.014$ & 3.882 & 5631.8 & 1450.6 & 5016 \\
$r$ & $+0.006 \pm 0.010$ & 2.654 & 3752.8 & 1414.1 & 6078 \\
$i$ & $-0.012 \pm 0.008$ & 1.657 & 2065.3 & 1246.2 & 7734 \\
$z$ & $-0.001 \pm 0.006$ & 1.195 & 1385.0 & 1158.6 & 9121 \\
\hline
\end{tabular}

$\epsilon_{\zeta}$ are those derived from Figure 2 and must be subtracted from SkyMapper photometry to reproduce the $\mathrm{AB}$ system. $B w(\lambda)$ is the bandwidth of the filters, whereas $G(\lambda)$ and $H(\lambda)$ are attributes necessary to derive monochromatic and in-band physical fluxes (see discussion in the Appendix). The spectrum of Vega has been adopted to compute the effective wavelength $\lambda_{\text {eff }}$. Note that while $\epsilon_{\zeta}$ are specific for DR1.1, $G(\lambda), H(\lambda), B w(\lambda)$ and $\lambda_{\text {eff }}$ are valid for any future SkyMapper release (unless filter transmission curves are revised).

values of $\log g$, and $[\mathrm{Fe} / \mathrm{H}]$, observed magnitudes (and reddening if present) to derive $T_{\text {eff }}$ via Eq. (3). Converting observed magnitudes into fluxes introduces the dependence on photometric zero-points, and link them to a physical quantity such as the stellar effective temperature. The dependence on synthetic stellar fluxes is needed to derive bolometric corrections, but besides this, at no point we make use of theoretical predictions between magnitudes and colours. Empirical colour- $T_{\text {eff }}$ relations can be easily derived from the IRFM, as we do later on in Section 5.1.1. Once these relations are available, one could use them to link photometric zero-points to stellar effective temperatures bypassing the IRFM. While viable, we have not explored this approach, as it would introduce the extra ladder of building these relations. 

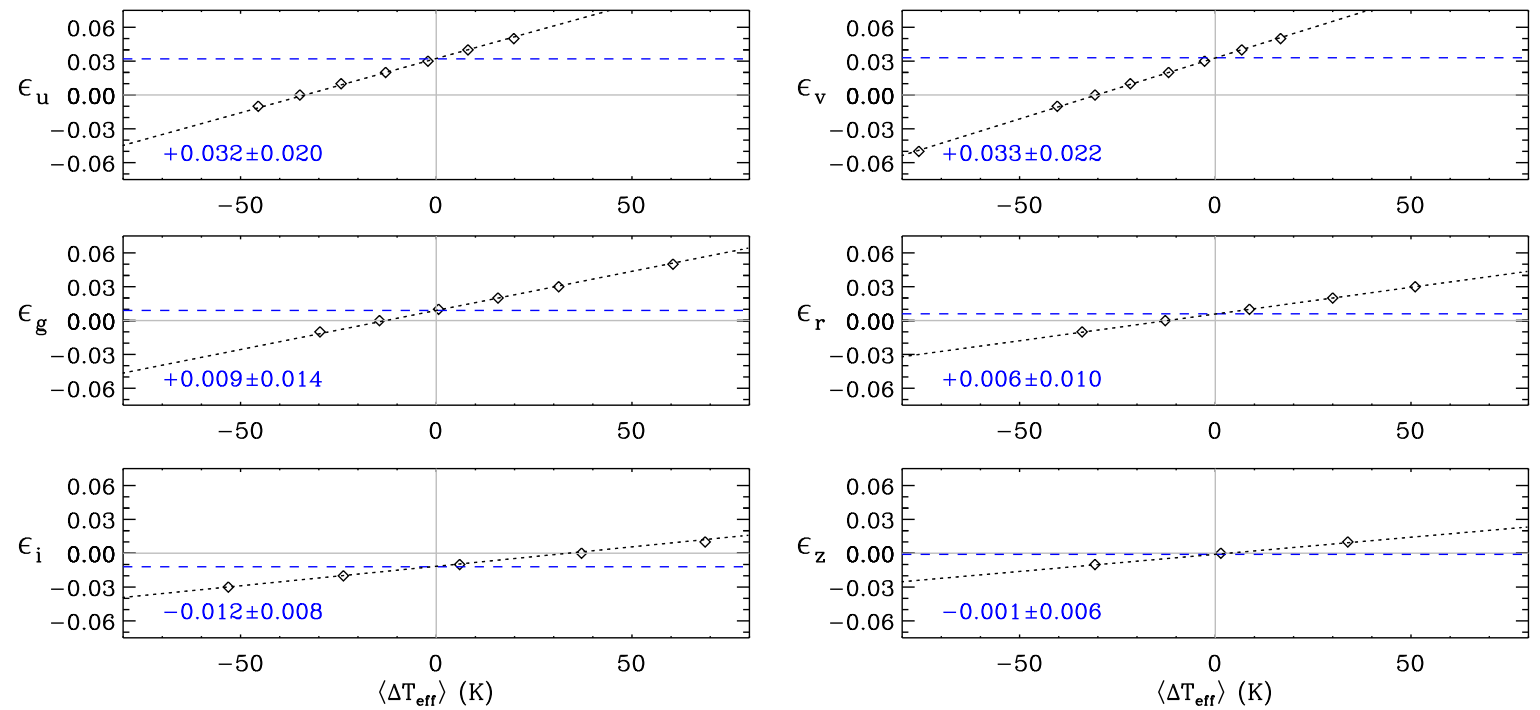

Figure 2. Photometric zero-points determined via the effective temperature scale. Diamonds are the weighted average of the effective temperature difference (SkyMapper-Reference sample) when SkyMapper zero-points are varied in the IRFM. Dotted lines are linear fits to the points. The adopted $\epsilon_{\zeta}$ (dashed blue lines) are determined from the intersection of the dotted lines with $\left\langle\Delta T_{\text {eff }}\right\rangle=0$, and they are indicated at the bottom of each panel. See text for further details.

\subsection{Reference Sample}

As we have explained in the previous Section, in order to infer the SkyMapper DR1.1 photometric zero-points we need a sample of stars for which we accurately know their $T_{\text {eff }}$. To this purpose we use stars from Casagrande et al. (2010, 2011) whose effective temperatures were homogeneously determined via the IRFM, and for which the uncertainty on the zero-point of the $T_{\text {eff }}$ scale is of order $20 \mathrm{~K}$. This accuracy implies that we are able to pin down photometric zero-points to about $0.01 \mathrm{mag}$. In order for this exercise to be entirely differential in $T_{\text {eff }}$, for each star we adopt parameters identical to Casagrande et al. (2010, 2011), i.e. the same [Fe/H], $\log g$, 2MASS photometry, and reddening (usually absent, or very small due to the nearby nature of the sample). We also remark that for our purposes it is essential to have stars from a well controlled sample, or systematic differences between heterogeneous $T_{\text {eff }}$ scales (e.g., using literature compilations) would dominate over the zero-point effects we wish to determine. Crucially, the zero-point of the effective temperature scale will impact the absolute flux scale, and hence the $\epsilon_{\zeta}$ we derive. Stars with reliably measured angular diameters would provide an equally good reference set (e.g. Karovicova et al. 2018, White et al. 2018), but only a handful of such objects are presently available, and because of their brightness they are also saturated in SkyMapper. We remark that the $T_{\text {eff }}$ scale we adopt has been tested against interferometric angular diameters confirming its accuracy (Casagrande et al. 2014a; Karovicova et al. 2018, White et al. 2018).

We find a total of 544 stars having a measurement in at least one SkyMapper band, and effective temperatures from Casagrande et al. (2010, 2011, which we refer to as the "Reference sample"). When $T_{\text {eff }}$ are determined implementing SkyMapper photometry into the IRFM, we refer to the same stars as the "SkyMapper sample". While nearly all 544 stars in this sample have $u v$ photometry, only a small percentage have griz measurements the number of available stars in these passbands varies between 19 and 32. This is due to the fact that stars in Casagrande et al. (2010. 2011) are quite bright, and the saturation limit for griz is brighter than for $u v$ magnitudes.

\section{2 uvgriz zero-point determination}

We implement the IRFM using one SkyMapper band at the time (in addition to 2MASS, which is always used), and vary its $\epsilon_{\zeta}$ across a suitable range, until on average stars in the SkyMapper sample have the same $T_{\text {eff }}$ as in the Reference sample i.e., we reproduce the zero-point of our adopted temperature scale. This is done computing $\left\langle\Delta T_{\text {eff }}\right\rangle$, which is the weighted average of the effective temperature difference between the SkyMapper and the Reference sample. For stars in both samples, weights are given by internal $T_{\text {eff }}$ uncertainties: we run a Monte Carlo simulation into the IRFM to assess the degree to which effective temperatures are affected by the photometric uncertainties in the input data. For photometric errors beyond 0.04 mag in griz, we note a slight correlation with $\Delta T_{\text {eff }}$, whereas we do not see any for $u v$ bands (whose maximum photometric errors are around $0.1 \mathrm{mag}$ ). Hence, when computing $\left\langle\Delta T_{\text {eff }}\right\rangle$ we exclude stars with errors larger than the values quoted above. We also apply a $3 \sigma$ clipping to remove stars with large effective temperature differences, and we track down the reason of those in the next Section.

The zero-point of the SkyMapper $T_{\text {eff }}$ scale varies linearly with the value assumed for each $\epsilon_{\zeta}$ into the IRFM. This means that the correct value to adopt for $\epsilon_{\zeta}$ can be determined by a linear fit intersecting an average effective temperature difference of zero. This is shown in Figure 2 and the zero-points so derived are reported in Table 1 Uncertainties are obtained by adding to the uncertainty of the intercept, the systematic if the reference $T_{\text {eff }}$ scale were to be shifted by $\pm 20 \mathrm{~K}$ (which is the zero-point uncertainty of the Reference sample). We remark that the zero-points we determine in this way are usually in good agreement with those obtained from the CALSPEC spectrophotometry. The largest discrepancy is only $1.4 \sigma$, and the sign of the zero-points agrees for all, but $r$ band (compare Figure 1 with Figure 2,

The zero-points in Table 1 must be subtracted from the SkyMapper DR1.1 magnitudes if one wishes to place them onto the $\mathrm{AB}$ system (or conversely, they must be added to the $\mathrm{AB}$ definition to replicate SkyMapper DR1.1 magnitudes). Importantly, these 

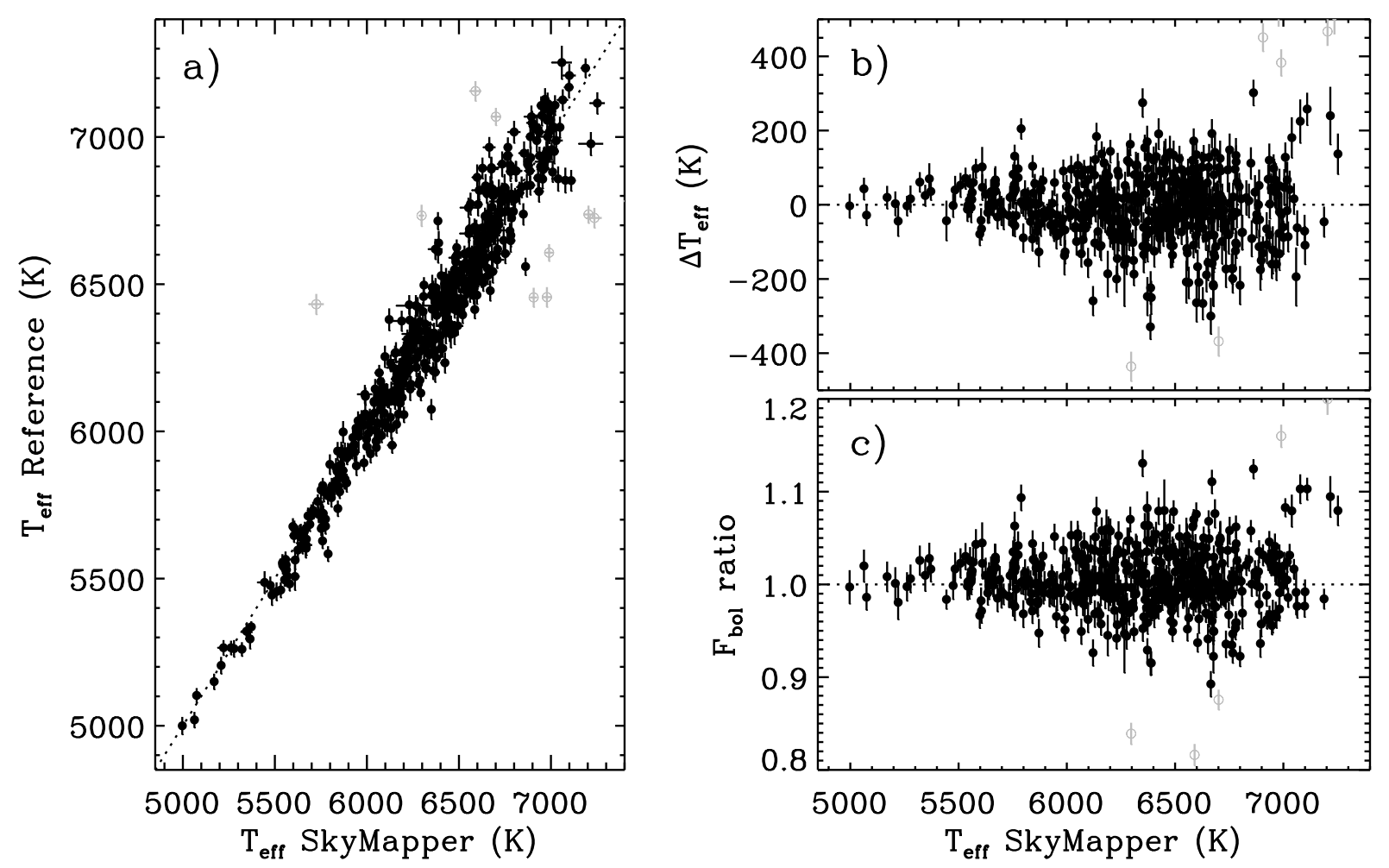

Figure 3. Panel a): comparison between the effective temperatures obtained implementing SkyMapper photometry into the IRFM (with zero-points reported in Table 1), and the Reference sample of Casagrande et al. (2010, 2011). Panel b): effective temperature difference (SkyMapper-Reference). Panel c): relative difference in bolometric flux (SkyMapper/Reference) for the same stars. Error bars are internal uncertainties obtained from a Monte Carlo simulation on photometric errors. For each star we use as many SkyMapper bands as possible, depending on quality flags and photometric errors. Stars marked in grey have been removed with a $3 \sigma$ clipping. See text for details.

zero-points are global. We discuss in the next Section their dependence (or lack thereof) on sky-position and magnitudes.

With the zero-points appropriate for each uvgriz filter, we can then apply the IRFM using as many SkyMapper bands as possible. Figure 3 confirms that when using more SkyMapper bands in the IRFM we still reproduce the effective temperature scale of the Reference sample (as one would expect), the weighted difference being $0 \pm 2 \mathrm{~K}$, with an rms of $88 \mathrm{~K}$. There are some clear outliers, which stem from spatial variations of zero-points across the sky (see Section 3.3. Notice that although we have discussed everything in terms of $T_{\text {eff }}$, by changing the SkyMapper zero-points we are also able to reproduce on average the same bolometric fluxes (and thus angular diameters) of the Reference sample; the weighted ratio of bolometric fluxes agrees to $0.5 \pm 0.1$, with a 3 percent rms. (Figure 3k). The above differences would be $-31 \mathrm{~K}$ and -0.9 percent in flux if no zero-points were applied (i.e., wrongly assuming perfect standardization to the $\mathrm{AB}$ system) and $-5 \mathrm{~K}$ and 0.25 percent in flux if using the zero-points determined from the CALSPEC spectrophotometry.

\subsection{Spatial dependence of SkyMapper zero-points}

Ideally, photometric zero-points should be the same for all stars in the sky, independently of anything else. However, there can be a number of reasons why this assumption breaks down (see e.g., Stetson 2005, for a sobering discussion on the difficulty of standardizing observations). The method presented in Section 3 to determine SkyMapper zero-points has the advantage that it can be applied to a large sample of stars (instead of the handful having CALSPEC spectrophotometry), and thus it can be used to explore the dependence of photometric zero-points on various parameters. This is done in Figure 4. which shows the effective temperature difference (SkyMapper-Reference) when applying the zero-points of Table 1. and running one SkyMapper band at the time in the IRFM.

While only a handful of points are available for griz bands, no obvious trends can be found. Using the linear mapping of Figure 2 between zero-point shifts and $\Delta T_{\text {eff }}$, we convert the $\sim 0.03$ magnitude scatter reported by Wolf et al. (2018) for griz filters into an effective temperature scatter (grey bands in Figure 4). Most of the points are consistent with the location of the grey bands, thus confirming the conclusion of Wolf et al. (2018). However, large scatter and spatial trends are observed for $u$ and $v$ band, suggesting that the zero-points of those two bands are not standardized as well as for the other SkyMapper filters. This is not entirely unexpected, considered that SkyMapper does not observe $u v$ standards.

These trends are very clear as function of Galactic latitude $b$, although they also appear in Galactic longitude, RA and declination because of correlation among coordinates. To try understanding their origin, we briefly recall how the photometric calibration is achieved in SkyMapper, and refer to Wolf et al. (2018) for further details. Instead of using standard stars, photometric zero-points to standardize instrumental $u$ and $v$ magnitudes are estimated using transformations which involve APASS $g$ magnitudes, a dereddened colour term, and a reddening estimate. The dereddened colour term comes from converting APASS magnitudes into Pan-STARRS1, and then a linear Pan-STARRS1 to SkyMapper relation derived 

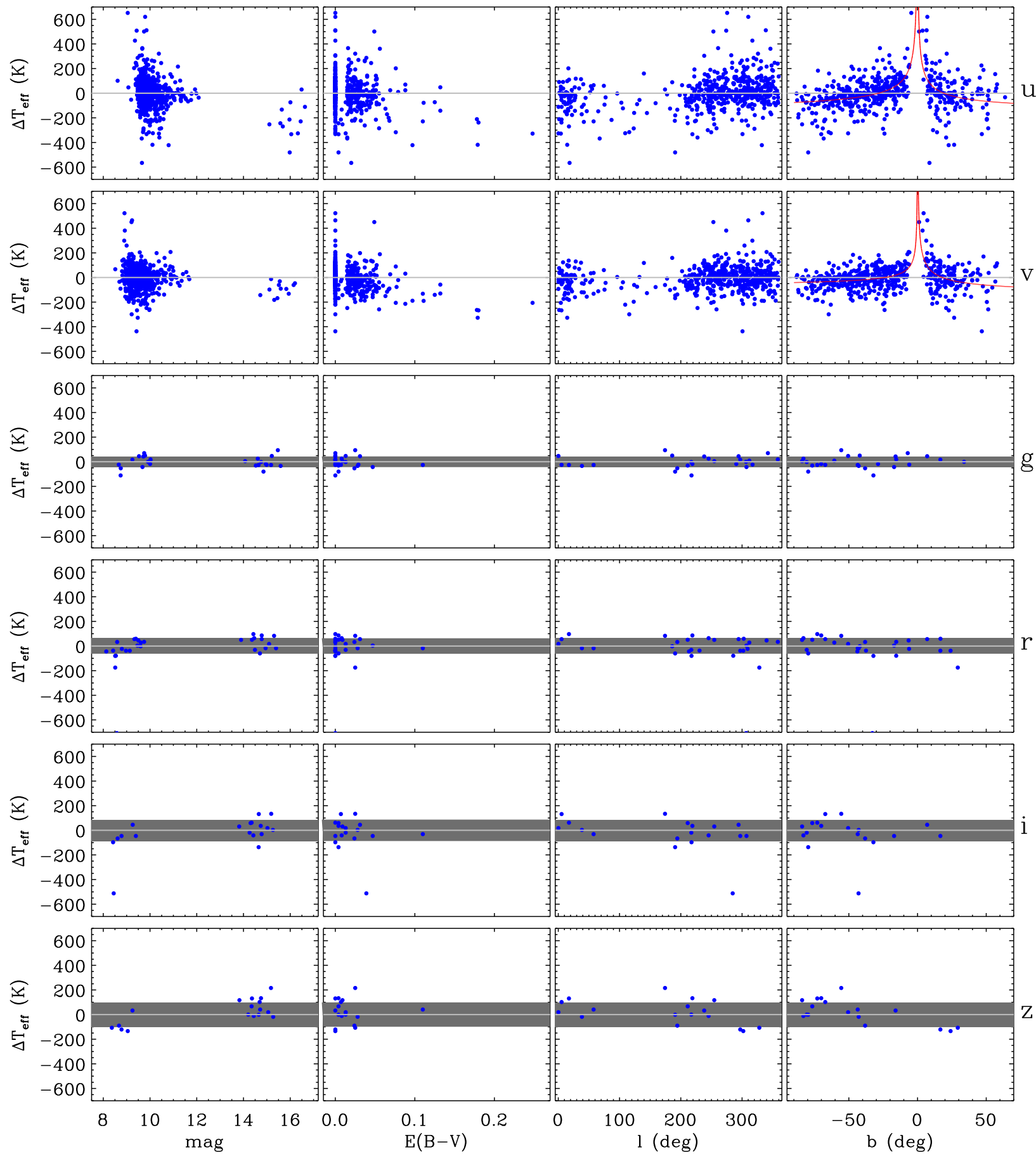

Figure 4. From top to bottom: $\Delta T_{\text {eff }}$ (SkyMapper-Reference) for uvgriz bands, as function of magnitudes, reddening, Galactic longitude $(l)$ and latitude $(b)$. Dark grey areas correspond to $T_{\text {eff }}$ variations of 0.03 mag in griz, which amount to the scatter and mean offset reported for those bands by Wolf et al. (2018).

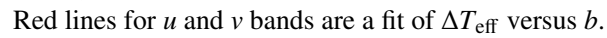

from unreddened stellar templates. The reddening estimate is based on a rescaling of the Schlegel et al. (1998) map. This procedure defines the average zero-points for each frame. The actual zeropoints applied to each star come from fitting the differences in the predicted (from the above procedure) and instrumental magnitudes for each star as function of spatial position on the CCDs. This is done to take into account atmospheric extinction gradients across the large field of view of the SkyMapper telescope. This approach proves to work remarkably well for griz bands, as confirmed by the 2.3 percent scatter (and up to 1 percent mean offset) for stars in common between Pan-STARRS1 and calibrated SkyMapper magnitudes (Wolf et al. 2018 see). However, a higher scatter is to be expected in $u v$ bands, because of their stronger sensitivity to stellar parameters, and reddening. In fact, the above procedure to predict 

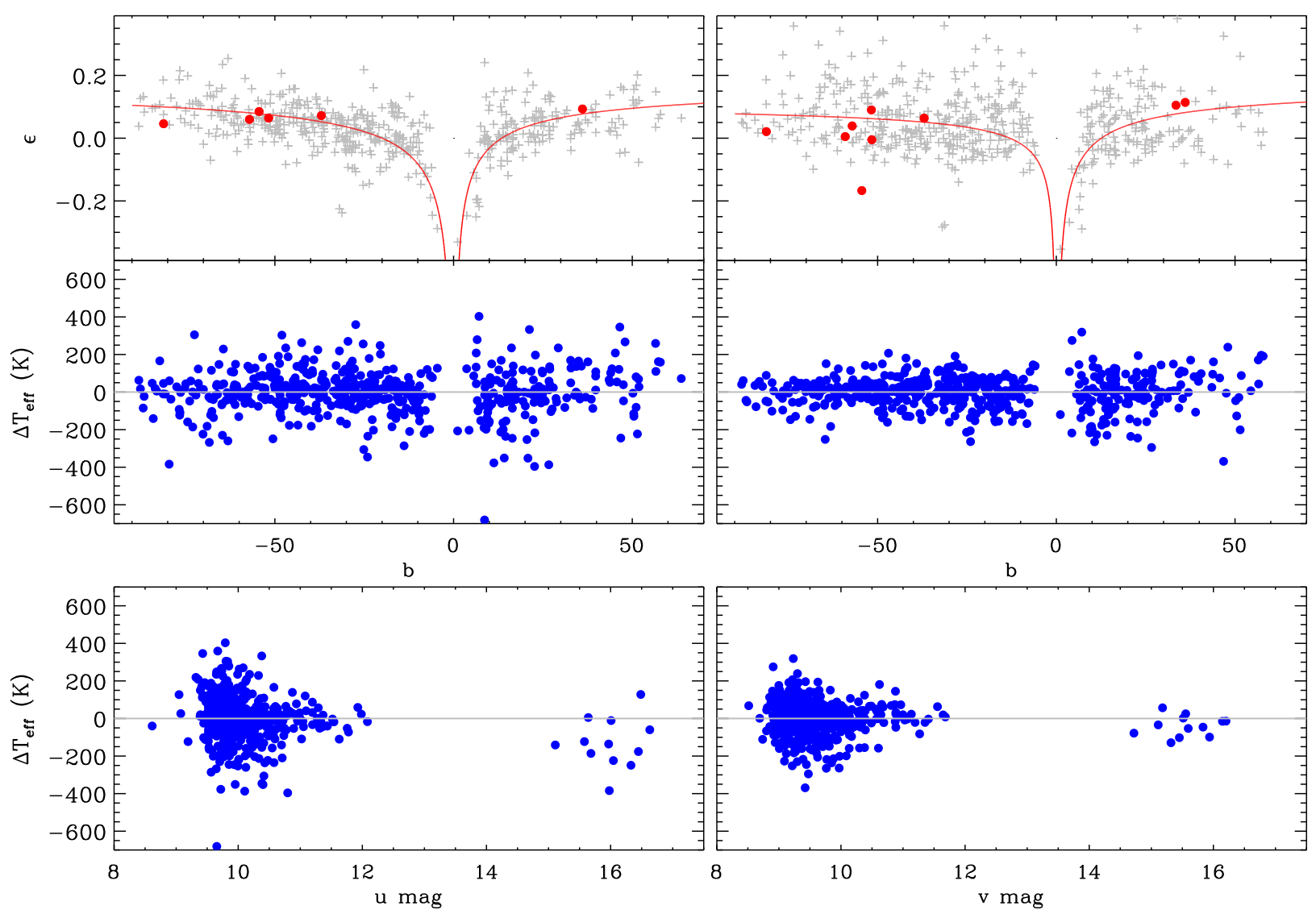

Figure 5. Top panels: continuous line shows the $u$ (left) and $v$ (right) zero-point dependence on Galactic latitude $(b)$, as per Eq. (4) and (5). Filled circles are the observed minus AB magnitudes for CALSPEC stars. Crosses are the difference between SkyMapper and Strömgren $u$ (left) and $v$ (right) magnitudes, as explained in the text. Middle and lower panels show $\Delta T_{\text {eff }}$ (SkyMapper-Reference) as function of $b$ and magnitudes, after correcting SkyMapper zero-points.

$u v$ magnitudes for a given star has a dispersion of order 0.1 mag or more. However, assuming only random errors, the formal uncertainty on the $u v$ zero-points is often well below $0.01 \mathrm{mag}$, because zero-points are typically determined using several hundred stars in each frame. Nevertheless, Figure 4 suggests that the quality of $u v$ magnitudes is substantially poorer than the percent level achieved for griz. The strong $\Delta T_{\text {eff }}$ trend as function of Galactic latitude likely stems from the reddening prescriptions adopted to calibrate SkyMapper magnitudes, as described above. In fact, $\Delta T_{\text {eff }}$ grows positive and larger closer to the plane, meaning that $T_{\text {eff }}(u v$ magnitudes) in SkyMapper are overestimated (too bright) close to the plane, and vice versa at high Galactic latitudes. Since we adopt the same reddening for the Reference and the SkyMapper sample (and reddening for these stars is typically very low, see discussion in Section 3.1) this can only mean that the $u v$ zero-points adopted to standardize DR1.1 magnitudes are overcorrected for reddening close to the plane, and vice versa at high latitudes.

In Figure 4 we fit $\Delta T_{\text {eff }}$ as function of $1 / \sqrt{|b|}$, and use the mapping of Figure 2 to derive how zero-points vary across the sky. We obtain the following functional forms:

$$
\epsilon_{u}= \begin{cases}0.198-0.727 / \sqrt{b} & b>0^{\circ} \\ 0.198-0.886 / \sqrt{|b|} & b<0^{\circ}\end{cases}
$$

and

$$
\epsilon_{v}= \begin{cases}0.200-0.710 / \sqrt{b} & b>0^{\circ} \\ 0.125-0.451 / \sqrt{|b|} & b<0^{\circ}\end{cases}
$$

where these zero-points must be subtracted from SkyMapper magnitudes to reproduce the $\mathrm{AB}$ system, and $b$ is the Galactic latitude in degrees. The lines in the top panels of Figure 5 show the dependence of these zero-points on Galactic latitude. We also show the zero-points as traced by CALSPEC standards (filled circles), as well as the difference between SkyMapper and Strömgren $u$ and $v$ magnitudes for stars in the GCS (grey crosses). In comparing with Strömgren photometry, an arbitrary shift is applied to bring the grey crosses onto the continuous line, since Strömgren photometry is not onto the AB system. We remark that in no instance Strömgren $u$ and $v$ magnitudes were used to derive $T_{\text {eff }}$ for our stars, yet the same trend is found as function of Galactic latitude. This is particularly clear for $u$, where the SkyMapper and Strömgren transmission curves are nearly identical, whereas the SkyMapper $v$ band is shifted $\sim 200 \AA$ towards the blue compared to the Strömgren one. Applying our zero-point corrections to SkyMapper magnitudes removes the major trend in $\Delta T_{\text {eff }}$ versus $b$ (middle panels). The trends with $u$ and $v$ magnitudes seen in Figure 4 are also largely corrected for, and although not shown, the fit as function of $b$ is sufficient to remove the wobbling trends with Galactic longitude, as well as RA and declination. We have previously described how the $u v$ standardization is done in DR1.1, and pointed to reddening as the main cause for zero-point variations across the sky. Although other systematic effects might still remain, we prefer to have a minimum number of parameters in Eq. (4) and Eq. (5). Our fits remove the main trend as function of $b$, albeit in Figure $5 u$ and $v$ have still a 
scatter of $120 \mathrm{~K}$ and $90 \mathrm{~K}$, respectively (for comparison, the scatter in the SkyMapper other bands is between 50 and $100 \mathrm{~K}$ ). These translate to photometric uncertainties of order $0.1 \mathrm{mag}$ for $u$ and $v$. Interestingly though, the scatter when comparing $T_{\text {eff }}$ obtained implementing $u$ and $v$ band into the IRFM is much smaller, $65 \mathrm{~K}$, which implies an uncertainty of order $0.06 \mathrm{mag}$ in $u-v$. This likely indicates a degree of correlation between these two bands, which is not surprising given the similar standardization procedure in DR1.1 for the two filters.

Our proposed zero-point corrections amount to roughly $\pm 0.1 \mathrm{mag}$ across the sky, except for regions close to the Galactic plane. We remark that we have a handful of stars with $|b|<10^{\circ}$, and the high corrections returned at low latitudes should be used with caution at this stage. Also, $\epsilon_{u}$ and $\epsilon_{v}$ vary in similar fashion as function of $b$, thus giving further support to their correlation, and meaning that above $\sim 10^{\circ}$ from the plane, the $u-v$ index is affected by $\sim 0.06$ mag at most.

\subsection{Comparison to other methods for zero-points determination}

The method used here to improve photometric zero-points relies on stellar effective temperatures of a number of stars across the sky. In the literature there exist similar other methods, at least conceptually, where stellar properties are used to improve zero-points of large scale photometric surveys. One rather common technique uses the stellar-locus regression, where the stellar locus defined by stars in various colour-colour planes is assumed to be universal, and photometric zero-points in different frames are varied to match this assumed location (e.g., MacDonald et al. 2004, Ivezić et al. 2007, Covey et al. 2007, High et al. 2009). Another method is the stellar-colour regression, where stars with reasonably similar spectroscopic parameters are assumed to have same colours (Yuan et al. 2015). The pros and cons of these methods are largely discussed in the above literature. Very briefly, the stellar-locus regression strongly relies on the assumption that stellar properties do not vary across the different populations observed by a large scale survey. Strictly speaking, this is not true, as stellar age and metallicity gradients are known to exist across the Galaxy (e.g. Boeche et al.2014, Casagrande et al. 2016, Ciucă et al. 2018). Hence, the stellar-locus regression is usually not applied to ultraviolet filters, which are intrinsically more sensitive to variations of stellar properties (High et al. 2009). Also, the stellar-locus regression necessarily correct for extinction, and it produces discrepant results if the sources of extincion vary significantly across a field of view. The stellar-colour regression requires instead the existence of a few photometrically well calibrated fields from which spectroscopic reference stars are selected in order to determine the intrinsic colours for a given set of stellar parameters. Stars with spectroscopic stellar parameters are then needed across the sky, and the reddening values of these stars must be known. While these limitations are real, they do not impede stellar-locus and stellar-colour regressions to achieve an internal precision of order 1 percent or better (e.g. High et al. 2009. Yuan et al. 2015).

The photometric standardization currently done in SkyMapper can also be regarded as a form of stellar-locus regression. In this case, the locus is defined by the stellar templates used to derive transformations from APASS to SkyMapper magnitudes (see summary in Section 3.3. and Wolf et al.2018 for full details). As previously discussed, this approach works remarkably well for SkyMapper optical filters, but not for the $u v$ ones because of their sensitivity to stellar parameters (a dependence which is not accounted for in the stellar-locus approach). The method we have developed in this paper aim to overcome this limitation, by varying photometric zeropoints until reference stellar effective temperatures from the IRFM are reproduced. The advantage is that the method is differential with respect to stellar properties and reddening: the same $\log g,[\mathrm{Fe} / \mathrm{H}]$ and $E(B-V)$ adopted to derive reference effective temperatures are used to implement SkyMapper photometry of the same stars into the IRFM. Also, the IRFM is only mildly sensitive to the assumed $\log g$ and $[\mathrm{Fe} / \mathrm{H}]$ of stars, and it readily allows to map known $T_{\text {eff }}$ into photometric zero-points. Correct zero-points can thus be derived if the absolute zero-point of the $T_{\text {eff }}$ scale is known. This last requirement limits the number of stars across the sky which can be used for this purpose.

\section{REDDENING COEFFICIENTS AND REDDENING FREE INDEXES}

A non-negligible amount of foreground dust is present for stars roughly beyond $\sim$ 70pc (e.g., Lallement et al. 2003). Since SkyMapper saturates around $g \sim 10$ (the exact value varying with seeing conditions), the above distance implies that sources with absolute magnitudes brighter than $M_{g} \sim 6$ will suffer from extinction. In other words, this affects all stars observed by SkyMapper, unless we limit ourselves to nearby dwarfs. In this Section we provide extinction coefficients suitable for late-type stars. Users can adopt those together with their preferred source of reddening estimates to unredden observed photometry, before applying the calibrations we provide in Section 5.1.1 and 5.1.3. We also lay out the formalism to use extinction coefficients to create reddening free pseudo-colours and -magnitudes.

Extinction is usually parametrized as a function of reddening $E(B-V)$, and $R_{V}$. The latter is the ratio of total to selective extinction in the optical, found to be $\simeq 3.1$ for most Galactic sightlines (e.g., Schlafly et al. 2016). It can be shown that a given $E(B-V)$ and $R_{V}$ will affect stars of different spectral types differently (e.g., Casagrande \& VandenBerg 2014). For example, the extinction coefficients reported in Wolf et al.(2018) are based on a flat spectrum, and the Fitzpatrick (1999) extinction law. In our implementation of the IRFM, we adopt the Cardelli et al. (1989)/O'Donnell (1994) extinction law, and iteratively compute extinction coefficients using a synthetic spectrum at the $T_{\text {eff }}, \log g$ and $[\mathrm{Fe} / \mathrm{H}]$ of each star to deredden them. In practical terms, extinction coefficients are rather constant, but for the bluest filters at the coolest $T_{\text {eff }}$. Extinction coefficients for the SkyMapper system are given in Table 2 Once extinction coefficients are known, unreddened magnitudes $m_{\zeta, 0}=m_{\zeta}-R_{\zeta} E(B-V)$ and colours $(\zeta-\eta)_{0}=(\zeta-\eta)-E(\zeta-\eta)$ $=(\zeta-\eta)-\left(R_{\zeta}-R_{\eta}\right) E(B-V)$ can be derived.

Reddening-free pseudo-colours $c_{P S}$ and pseudo-magnitudes $m_{P S}$ can also be built as follows:

$$
c_{P S}=(\zeta-\eta)-X(\xi-\ni)
$$

where $\zeta, \eta, \xi$ and $\ni$ are any combination of SkyMapper filters, and $X$ is a multiplicative factor such that any dependence on reddening cancels out. It can be easily proved that this conditions is met when:

$$
X=\frac{R_{\zeta}-R_{\eta}}{R_{\xi}-R_{\ni}} .
$$

Similarly, for pseudo-magnitudes:

$$
m_{P S}=\zeta-X(\eta-\xi)
$$



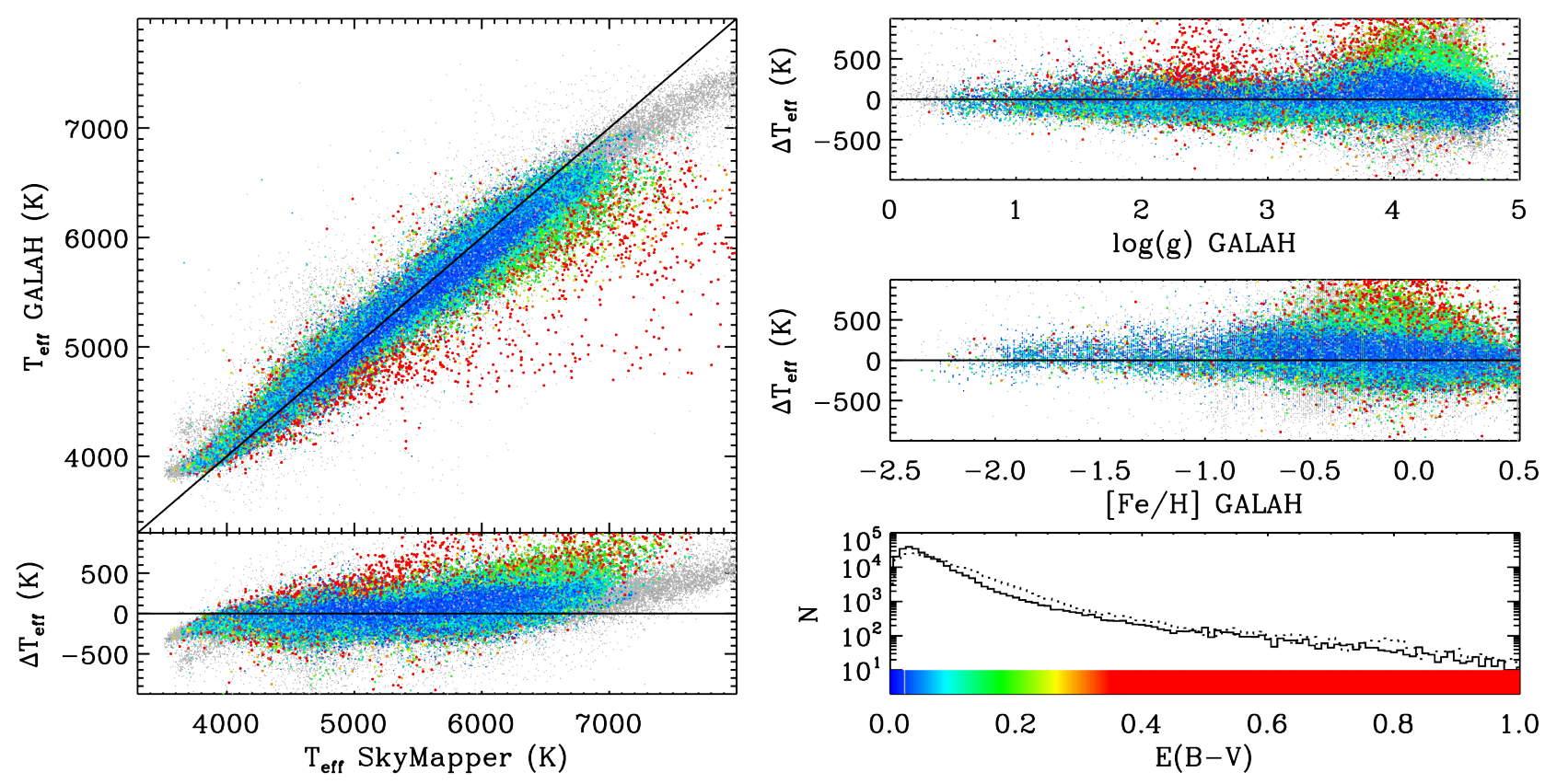

Figure 6. Comparison between $T_{\text {eff }}$ derived implementing SkyMapper photometry into the IRFM, and the GALAH spectroscopic survey (Buder et al. 2018). Residuals (SkyMapper-GALAH) are shown as function of stellar parameters, and colour coded by reddening according to the scale in the bottom right panel. Dotted histogram is reddening from Schlegel et al. (1998), while continuous histogram shows the rescaled values we adopt. Grey points are stars flagged as unreliable in GALAH.

Table 2. Extinction coefficients $R_{\zeta}$ for a solar temperature star. Notice that for a nominal $E(B-V)$, the excess in any given colour combination is $E(\zeta-$ $\eta)=\left(R_{\zeta}-R_{\eta}\right) E(B-V)$.

\begin{tabular}{cccccc}
\hline$R_{u}$ & $R_{v}$ & $R_{g}$ & $R_{r}$ & $R_{i}$ & $R_{z}$ \\
\hline 4.88 & 4.55 & 3.43 & 2.73 & 1.99 & 1.47 \\
\hline
\end{tabular}

$R_{u}$ has a strong dependence on $T_{\text {eff }}$, which can be fit as $R_{u}=4.95-2.6 \times$ $10^{21} T_{\text {eff }}^{-6}$. For $R_{g}=3.68-1471 \times T_{\text {eff }}^{-1}$. For the remaining filters, reddening coefficients vary less than $\sim 0.1$ over the range $3500 \mathrm{~K}<T_{\text {eff }}<10000 \mathrm{~K}$ explored in this work. The values reported here agree with the fit at the solar value from Table B1 of Casagrande \& VandenBerg (2018b where the fit for $u$ and $g$ band is valid on a much smaller $T_{\text {eff }}$ range).

where

$$
X=\frac{R_{\zeta}}{R_{\eta}-R_{\xi}} .
$$

It must be pointed out that the above reddening-free indices are meaningful only over the $T_{\text {eff }}$ regime where extinction coefficients are nearly constant. Also, we remark that the use of reddening-free indices is often a trade-off: in fact while they bypass the dependence on reddening, they correlate more poorly with stellar parameters.

\section{SKYMAPPER MEETS GALAH}

The GALactic Archaeology with HERMES (GALAH) is a stellar spectroscopic survey conducted on the Anglo-Australian Telescope (De Silva et al.2015). GALAH stellar parameters are obtained with "The Cannon" (Ness et al. 2015), a data-driven approach calibrated upon a training set that covers the FGK-type stars (see Buder et al. 2018 for further details). Currently, nearly half million stars have been observed and analyzed, with over 270000 spectra in common with SkyMapper DR1.1. Here, we apply the IRFM on all these stars, and check the performance of data-driven $T_{\text {eff }}$ determination in GALAH DR2 (Buder et al. 2018).

Apart from a few pointings along the plane, nearly all of the SkyMapper $\cap$ GALAH targets have Galactic latitudes $|b|>10^{\circ}$, meaning that the most obscured and patchy region of the Galactic plane are avoided. Yet, reddening can have a non-negligible contribution, and must be taken into account in photometric methods. For each target we rescale $E(B-V)$ from Schlegel et al. (1998) using the same procedure developed for RAVE DR5 (Kunder et al.2017), and which is solely based on the intrinsic colour of red clump stars (as described in more details in Casagrande et al.2014b).

We implement the IRFM exploring different combinations of the photometric zero-points derived in the previous Section. Because of the zero-point spatial variations affecting $u$ and $v$ bands, and their small flux contribution (when other SkyMapper bands are also implemented), we adopt $T_{\text {eff }}$ derived using only grizJHK $K_{S}$ in the IRFM. Notice however that we also derived temperatures including $u v$ photometry as a check, and verified the effect to be rather minor. The mean difference and scatter is of order few Kelvin, and $30 \mathrm{~K}$ respectively, either using the constant zero-points from Table 11. or the spatially dependent ones from Eq. (4) and (5).

Figure 6 shows the comparison between $T_{\text {eff }}$ from the IRFM and GALAH, colour-coded by the adopted $E(B-V)$. For low reddening regions, the agreement is usually excellent across the entire stellar parameter range, and it degrades in regions of high extinction, where $T_{\text {eff }}$ from the IRFM are typically hotter (thus, indicating that in these regions reddening is still preferentially overestimated, despite our rescaling). Spectra labelled as unreliable in GALAH (data reduction or Cannon flags $\neq 0$ ) are plotted in grey. Effectively all of the stars above $7000 \mathrm{~K}$ are flagged in GALAH, because of the lack of a training set in this regime, forcing the data-driven approach to extrapolate the determination of stellar parameters. The 

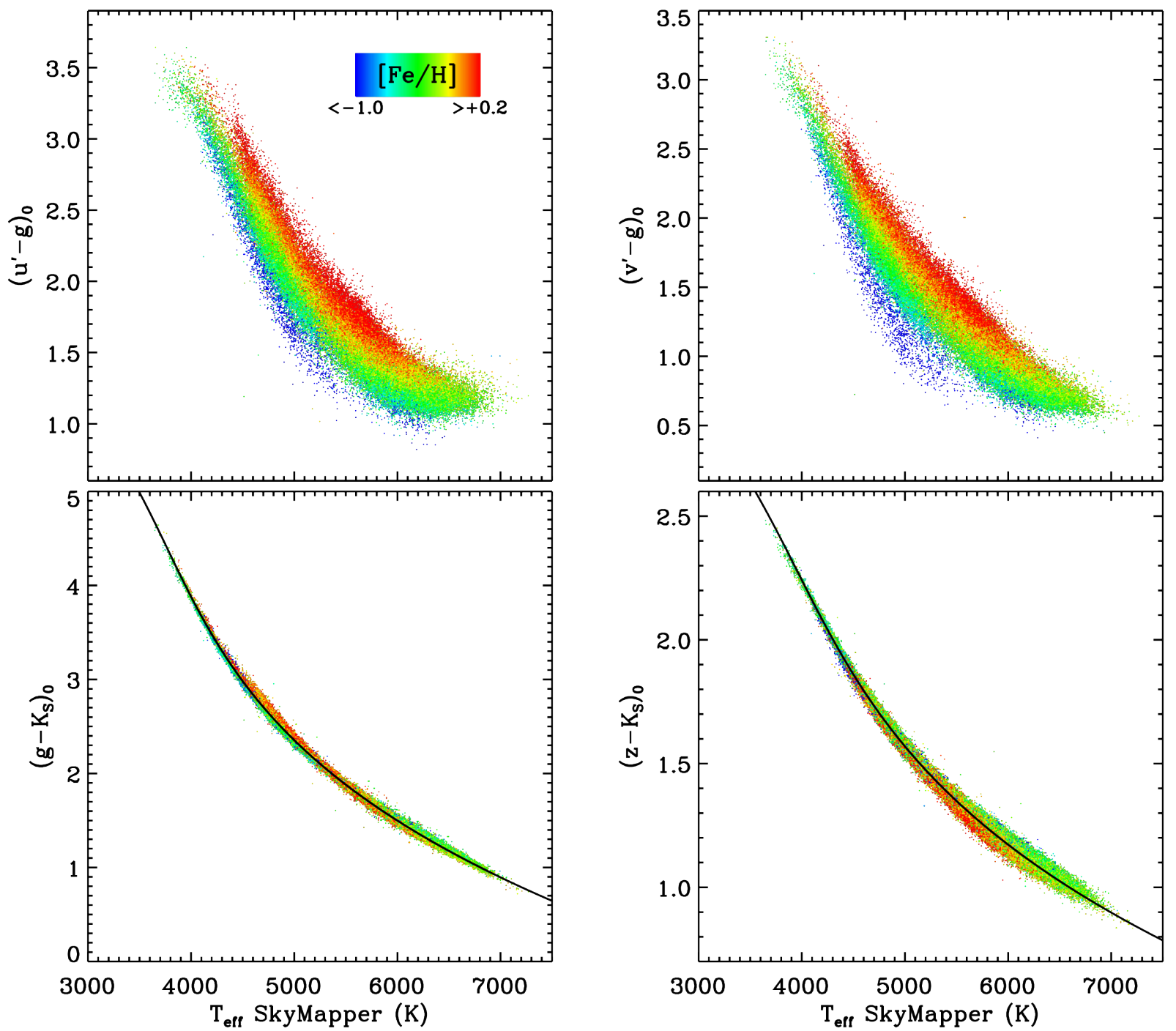

Figure 7. Colour-temperature relations for a few combinations of SkyMapper and 2MASS filters. See Section 5.1 for the definition of $u^{\prime}$ and $v^{\prime}$. All colours are dereddened using $E(B-V)$ as described in the text. Only stars with $E(B-V)<0.05$ are shown. Stars are colour-coded according to their GALAH metallicity, with the scheme indicated in the inset on the upper left panel. For $[\mathrm{Fe} / \mathrm{H}]<-1.0 \mathrm{dex}(>0.2 \mathrm{dex})$ the colour is kept fixed to blue (red). Eq. (10) and [11) are shown as continuous lines in the bottom panels.

IRFM indicates that the data driven-approach underestimates effective temperatures in this regime, saturating at $8000 \mathrm{~K}$ which is the limit of the grid of model atmospheres used for the training set (we checked that this trend is not an artefact of stars affected by high values of extinction). This comparison shows how well calibrated effective temperatures from the IRFM can be helpful to improve spectroscopic pipelines.

After removing flagged spectra, the SkyMapper-GALAH mean (median) $\Delta T_{\text {eff }}$ is $61 \mathrm{~K}(49 \mathrm{~K})$ with a scatter of $183 \mathrm{~K}$ when stars are considered irrespectively of their reddening. The above numbers reduce to $\Delta T_{\text {eff }}=51 \mathrm{~K}(50 \mathrm{~K})$ with a scatter of $132 \mathrm{~K}$ when restricting to $E(B-V)<0.10$, and $\Delta T_{\mathrm{eff}}=12 \mathrm{~K}(12 \mathrm{~K})$ with a scatter of $123 \mathrm{~K}$ for $E(B-V)<0.01$. This suggests that reddening can easily introduce systematics of order of a few tens of $\mathrm{K}$ on the zero-point of the $T_{\text {eff }}$ scale, and it is the primary source of uncertainty rather than the photometric zero-points when deter- mining $T_{\text {eff }}$. Further below we explore the sensitivity of SkyMapper colours to $[\mathrm{Fe} / \mathrm{H}]$ and $\log g$ from GALAH, and $T_{\text {eff }}$ from the IRFM.

\subsection{The sensitivity of SkyMapper colours to stellar parameters}

For spectral types ranging from approximately $\mathrm{F}$ to early $\mathrm{M}$, we explore the dependence of SkyMapper colours on stellar parameters. For the latter we adopt $[\mathrm{Fe} / \mathrm{H}]$ and $\log g$ from the GALAH sample (using only non-flagged stars), whereas effective temperatures come from the IRFM.

In all instances, colours have been dereddened with the $E(B-$ $V$ ) derived in Section 5 , and using extinction coefficients appropriate to the $T_{\text {eff }}, \log g$ and $[\mathrm{Fe} / \mathrm{H}]$ of each star (as discussed in Section 4). In the remainder of the paper, all plots and calibrations are corrected for reddening, and this is indicated by the 0 subscript. 

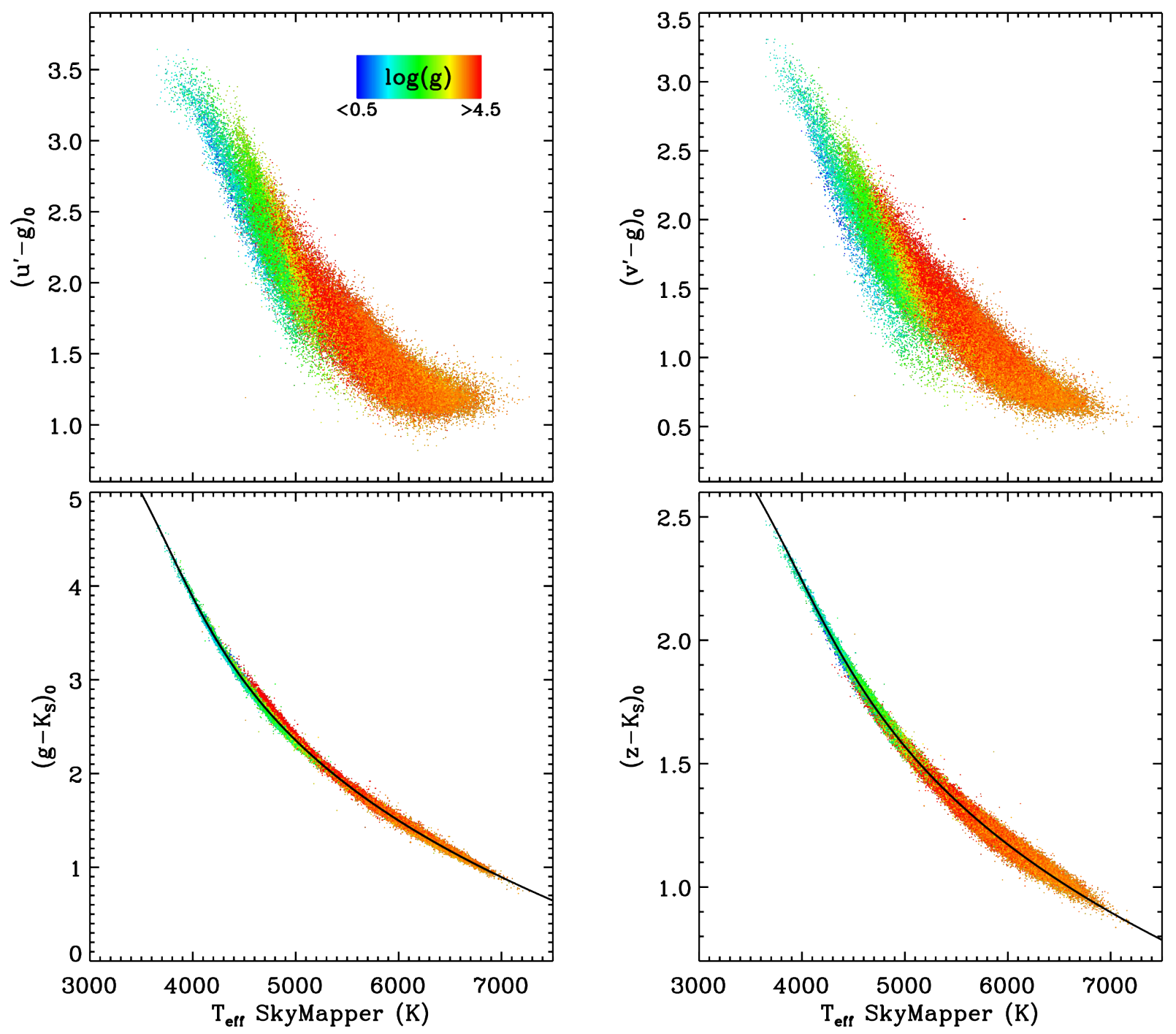

Figure 8. Same as previous figure, but with stars colour-coded according to their GALAH surface gravity. For $\log g<0.5$ dex ( $>4.5$ dex) the colour is kept fixed to blue (red).

Users should always correct for reddening their input photometry before applying our calibrations. Concerning the zero-points offsets discussed in Section 3 and 3.3 a few remarks are necessary. Constant zero-point offsets are of no importance when a calibration between observed colours and stellar parameters is built, since any zero-point is automatically factored into the calibration. Hence, zero-point corrections must not be applied to Eq. 10. and (11). On the contrary, spatially dependent zero-points must be corrected for, and this is the case for the metallicity calibration discussed further below. In the rest of the paper, we define $u^{\prime}=u-\epsilon_{u}$ and $v^{\prime}=v-\epsilon_{v}$, where $\epsilon_{u}$ and $\epsilon_{v}$ are given in Eq. 5.

\subsubsection{Colour $-T_{\text {eff }}$ relations}

$T_{\text {eff }}$ is the stellar parameter to which colours are most sensitive, and arguably the most needed e.g., to constrain spectroscopic analyses. Figure 7 and 8 show the colour- $T_{\text {eff }}$ relations derived from the IRFM in a selected number of colour indices, to highlight their dependence on metallicity and surface gravity. SkyMapper photometry performs satisfactorily to separate stars with different stellar parameters, in particular when using the $u$ and $v$ bands. From these figures, the interplay between metallicity and surface gravity in driving changes in photometric colours is obvious, besides sample selection effects (e.g., at the coolest $T_{\text {eff }}$ essentially all of the stars are giants, since $\mathrm{M}$ dwarfs are not analysed in GALAH). This means that is not straightforward to provide a unique functional form that works for all colour indices, and accounts at the same time for $\log g$ and $[\mathrm{Fe} / \mathrm{H}]$ effects. At the same time, in practical instances users are often interested to estimate $T_{\text {eff }}$ without prior knowledge of the metallicity and surface gravity of stars. We find that the $\left(g-K_{S}\right)_{0}$ and $\left(z-K_{S}\right)_{0}$ show a tight correlation with $T_{\text {eff }}$, and little sensitivity to $[\mathrm{Fe} / \mathrm{H}]$ and $\log g$. For these colours, third-order polynomials suffice to fit the data well:

$$
T_{\text {eff }}=9056.01-2732.89\left(g-K_{S}\right)_{0}+
$$




$$
522.40\left(g-K_{S}\right)_{0}^{2}-39.66\left(g-K_{S}\right)_{0}^{3}
$$

which has $\sigma=33 \mathrm{~K}$ and is valid for $0.46<\left(g-K_{S}\right)_{0}<4.65$, and

$$
\begin{aligned}
& T_{\text {eff }}=12884.70-9336.50\left(z-K_{S}\right)_{0}+ \\
& \quad 3567.35\left(z-K_{S}\right)_{0}^{2}-522.11\left(z-K_{S}\right)_{0}^{3}
\end{aligned}
$$

which has $\sigma=59 \mathrm{~K}$ and is valid for $0.68<\left(z-K_{S}\right)_{0}<2.48$.

\subsubsection{Surface gravity}

The gravity sensitivity of SkyMapper filters has already been explored in some detail in Wolf et al. (2018, see their figure 16 for examples of colour-colour plots discriminating dwarfs and giants). Here, we do not repeat that exercise, but rather focus on the gravity sensitivity of the $\left(v^{\prime}-g\right)_{0}$ vs. $\left(g-K_{S}\right)_{0}$ colours, which we will use as $[\mathrm{Fe} / \mathrm{H}]$ indicators in Section 5.1.3 Figure 9 shows the the dependence of this colour combination on $\log g$. We have already discussed how GALAH does not derive parameters for very cool dwarfs. Thus, in addition to the GALAH sample (colour-coded), we also include stars from RAVE DR5 (Kunder et al. 2017) here shown in grey), which has a larger number of late-type dwarfs and giants. For $\left(g-K_{S}\right)_{0} \gtrsim 3.5$ (which corresponds to $T_{\text {eff }} \lesssim 4200 \mathrm{~K}$ ), dwarf and giant stars clearly define distinct sequences in this colour plane. However, at bluer colours, there is very little dependence on $\log g$, and this is qualitatively confirmed by synthetic stellar colours (see the discussion in Casagrande \& VandenBerg 2014 2018b for the performance and limitation of stellar synthetic colours, in particular at blue wavelengths, and for cool stars).

\subsubsection{Colour $-[\mathrm{Fe} / \mathrm{H}]$ relation}

The determination of photometric metallicities is one of the goals behind the design of SkyMapper filters, in particular the $v$ band. In order to explore the correlation of different colour indices with stellar parameters we use Principal Component Analysis (PCA, e.g., Francis \& Wills 1999). Depending on the colour combination, we see the clear presence of up to three principal components. Regardless of the colour index though, the first component always correlates strongly with $T_{\text {eff }}$, while the second and the third correlate with different strength to $[\mathrm{Fe} / \mathrm{H}]$ and $\log g$, respectively. PCA thus confirms what we already knew, i.e., the colours of stars depend primarily on their effective temperature, while metallicity and surface gravity are less important, but non-negligible in certain bands (in fact, see Figure 7 and 8). However, the presence of a correlation does not guarantee that a useful calibration between stellar parameters and colour indices can always be found. For example, while all indices involving the $u$ band correlate with $\log g$, no calibration can be found beyond a qualitative separation between late-type dwarfs and giants. However, in the $\left(v^{\prime}-g\right)_{0}$ vs. $\left(g-K_{S}\right)_{0}$ colour plane we find a strong correlation between the second principal component and $[\mathrm{Fe} / \mathrm{H}]$. We derive the following calibration between colours and metallicities:

$$
[\mathrm{Fe} / \mathrm{H}]=\frac{-0.1815+0.1848\left(v^{\prime}-g\right)_{0}-0.1630\left(g-K_{S}\right)_{0}}{0.0649}+
$$

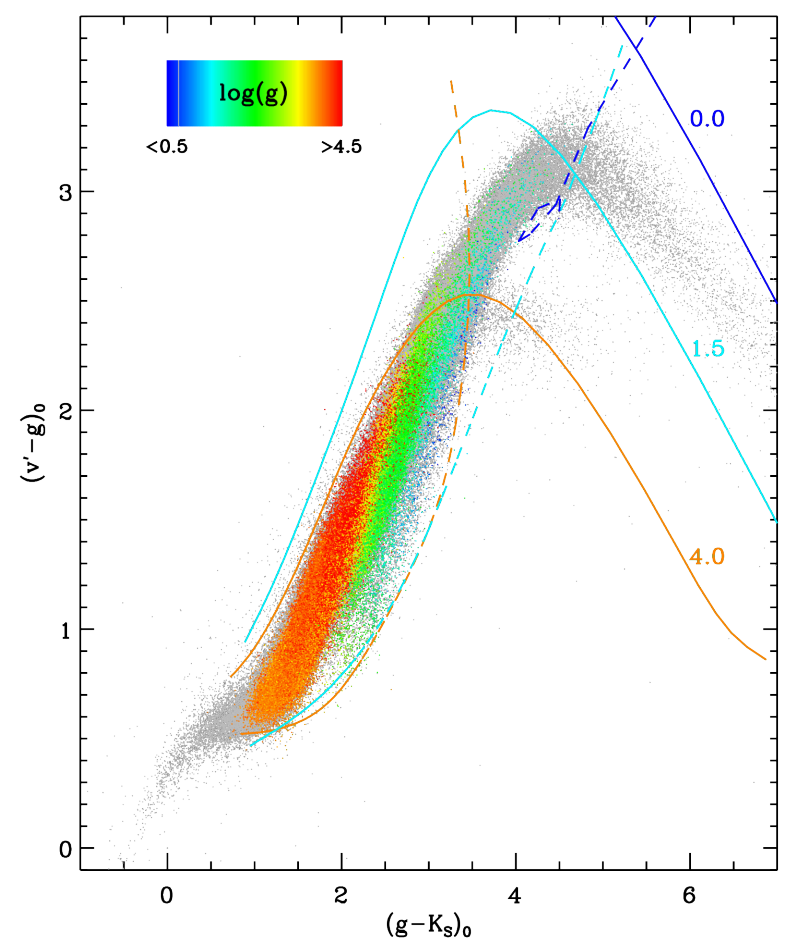

Figure 9. Colour-colour plane with GALAH stars coded by their $\log g$ as per inset panel. Grey dots are stars from RAVE DR5. Continuous lines are synthetic colours from Casagrande \& VandenBerg (2014 2018b) at the $\log g$ values indicated. Continuous and dotted lines are for $[\mathrm{Fe} / \mathrm{H}]=0.5$ and -4.0 , respectively.

where the first term is derived from PCA analysis, and the third order polynomial in $\left(g-K_{S}\right)_{0}$ is obtained fitting the residual as function of this colour index. We explored the use of more colour terms, as well as higher order polynomials, but found that our metallicity calibration did not improve. We suspect that this might be due to photometric uncertainties, where the gain of using more colours trades off with an increased error budget. Thus, we decide to adopt this rather minimalistic functional form, which also has the advantage of being broadly parallel to the reddening vector (see Figure 10). Our calibration is derived using only stars with $E(B-V)<0.05$, and located at Galactic latitudes $|b|>20^{\circ}$ to avoid introducing strong dependencies on zero-point corrections. We define two fiducial lines beyond which our calibration should not be extrapolated $P_{1}<\left(v^{\prime}-g\right)_{0}<P_{2}$ (grey lines in left-panel of Figure 12, where:

$P_{1}=1.3067-1.6731\left(g-K_{S}\right)_{0}+0.8129\left(g-K_{S}\right)_{0}^{2}-0.0810\left(g-K_{S}\right)_{0}^{3}$

and

$P_{2}=0.5783-0.0719\left(g-K_{S}\right)_{0}+0.4624\left(g-K_{S}\right)_{0}^{2}-0.0691\left(g-K_{S}\right)_{0}^{3}$.

With these criteria, our training sample comprises over 70,000 stars, and the standard deviation of our metallicity calibration is 0.21 dex. Also, these fiducials limit the metallicity range of our calibration, which applies down to $[\mathrm{Fe} / \mathrm{H}] \simeq-2$. We have verified that extending our calibration to more metal poor stars leads to mixed results. There are several reasons for this: while model atmosphere fluxes and isochrones indicate that Skymapper $v-g$ related colors should be useful down to metallicities of -4 , current photometric errors in SkyMapper ultraviolet bands (see discussion 



Figure 10. Left-panel: colour-colour plane with GALAH stars coded by their $[\mathrm{Fe} / \mathrm{H}]$ as per inset panel on the right. Grey lines define the boundary of our metallicity calibration, while continuous coloured lines trace Eq. [12) at indicated values of $[\mathrm{Fe} / \mathrm{H}]$. The arrow shows the direction of the reddening vector with length corresponding to $E(B-V)=0.1$. Right-panel: Kiel diagram for the same stars. In both panels, only stars with $E(B-V)<0.05$ and $|b|>20^{\circ}$ are shown, although relaxing these conditions does not qualitatively change the plots.

in Section 3.3 prevent to exploit its full potential to reliably single out the most metal poor stars. In addition, because of the increasing fraction of carbon-enhanced stars below -2 (e.g., Yong et al. 2013), the $\mathrm{B}-\mathrm{X}$ band of the $\mathrm{CH}$ molecules dump the flux around the location of the $v$ band ${ }^{4}$, hence mimicking a higher metal content. For a detailed investigation of the performances of SkyMapper photometry to identify extremely metal-poor stars, where other filter combinations are more appropriate, we refer to Da Costa et al. (2018).

Figure 11 compares the metallicities derived from Eq. (12) against the entire GALAH sample, irrespective of reddening and Galactic latitude, thus comprising over 160,000 stars. The standard deviation is virtually unchanged, $0.22 \mathrm{dex}$, confirming that reddening has a minimal impact upon our calibration. Also, the fact that we now probe latitudes closer to the plane, and still obtain satisfactory metallicities speaks well of our zero-point corrections. The most discrepant points in Figure 11 are indeed those with the highest reddening, but a large value of reddening does not univocally imply that photometric metallicities are unreliable. There is a large number of stars at high $E(B-V)$, for which spectroscopic and photometric metallicities are in good agreement (although in Figure 11 they are hidden behind an overwhelming number of stars at low reddening). Taking into account that GALAH metallicities

${ }^{4}$ We remark however that the CH G-band (A-X) falls within the $g$ filter and does not contaminate the Skymapper $v$ band. are precise to within $0.1 \mathrm{dex}$, this gives us confidence that photometric metallicities can be derived to a precision of 0.2 dex from our calibration. Residuals as function of colour, $T_{\text {eff }}$ and $\log g$ show that photometric metallicities are good across the entire parameter space explored, with increasing scatter and a mild offset towards the highest and lowest gravities, respectively. Although our metallicity calibration works well for both dwarfs and giants over the parameter space explored, we remark that GALAH does not provide parameters for dwarfs with $T_{\text {eff }} \lesssim 4500 \mathrm{~K}$ (see Kiel diagram in Figure 10 , where we expect to see a bifurcation between the dwarf and the giant sequence (Figure 9). Thus, the decreasing scatter in the residuals towards the reddest colours (and coolest $T_{\text {eff }}$ ) carries a sample selection effects: it reflects the adequacy of the calibration for cool giants, but it does not warrant its use for cool dwarfs. The fiducial $P_{1}$ is intended to limit contamination from cool dwarfs, although it does not remove them entirely. Thus, we advise using Gaia parallaxes to exclude cool dwarfs (see next Section), as well as to preferentially apply our metallicity calibration for $\left(g-K_{S}\right)_{0} \lesssim 3.5$, where the effect of surface gravity on the metallicity calibration is minor.

Finally, Figure 12 shows the residual of photometric versus spectroscopic metallicities as function of Galactic latitude. No trend is seen when $v^{\prime}$ magnitudes are used for the metallicity calibration, whereas this is not the case for $v$ : a clear trend appears as function of $b$, and this could e.g., lead to biases when measuring vertical metallicity gradients. We remark that the metallicity calibration is obtained using only stars at $|b|>20^{\circ}$, as well as with an entirely different sample (and method) than the one used to study 

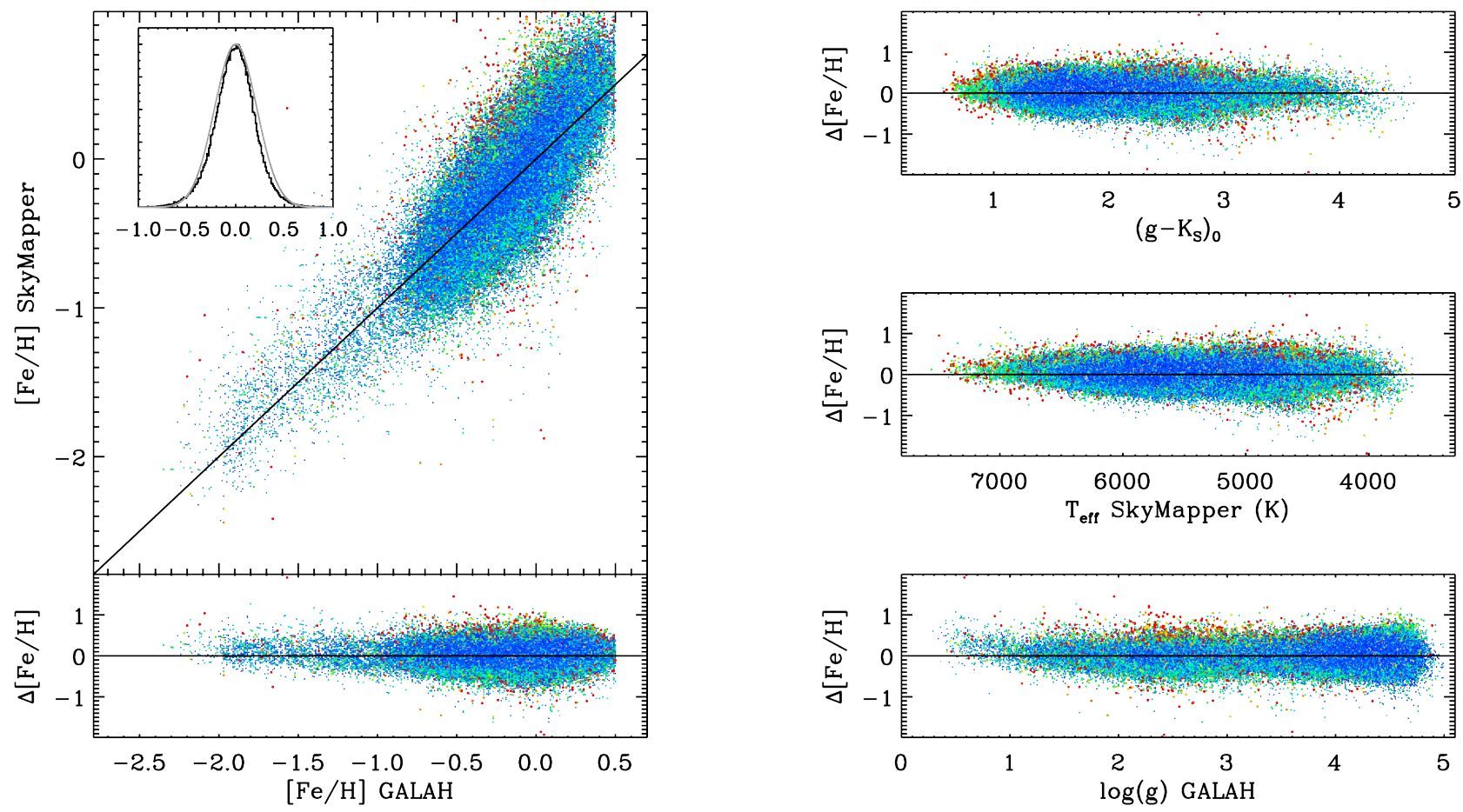

Figure 11. Left-panels: GALAH versus SkyMapper photometric metallicities for over 160,000 stars using our calibration (top), and residuals (SkyMapper-GALAH) fitted with a Gaussian of width 0.22 dex overplot (inset), and as function of [Fe/H] (bottom). Right-panels: residuals as function of $\left(g-K_{S}\right)_{0}, T_{\text {eff }}$ and $\log g$. In all figures, stars are colour-coded according to their reddening, as per the palette in lower-right panel of Figure 6

the spatial variation of zero-points (Section 3.3. The fact that a trend as function of $b$ is now seen in Figure 12 when we do not correct $v$ magnitudes, gives us further confidence that the zero-point variations we uncover are real.

\section{EXTERNAL VALIDATIONS \& COMPARISON TO SLOAN}

To further check the performance of our metallicity calibration, we first compare our photometric $[\mathrm{Fe} / \mathrm{H}]$ with two spectroscopic surveys other than GALAH, and then use SkyMapper+2MASS photometry to derive a metallicity map of the Milky Way. In all instances, we correct for reddening with the same prescription of Section 5 Finally we discuss the sensitivity to metallicity of the SkyMapper $v$ filter in comparison to the Sloan Digital Sky Survey (SDSS) $u$ band, the first survey to provide ultraviolet photometry for several million sources across the sky (Ivezić et al.2007).

The left-hand panel of Figure 13 compares our photometric metallicities against those in RAVE DR5 (Kunder et al. 2017). There is a mean offset of 0.09 dex (SkyMapper minus RAVE) and a scatter of $0.28 \mathrm{dex}$, which is consistent with the lower precision of $[\mathrm{Fe} / \mathrm{H}]$ in RAVE. The right-hand panel compares our metallicities against those in APOGEE DR14 (Abolfathi et al.|2018). In this case there is a smaller offset of -0.01 dex (SkyMapper minus APOGEE) and scatter of $0.25 \mathrm{dex}$. The advantage of these comparisons is the presence of cool dwarfs which are not part of the GALAH sample. Using Gaia's parallaxes (Gaia Collaboration et al. 2018), we clearly see that metallicity residuals deteriorate for $M_{g} \geqslant 7$, which we adopt as the absolute magnitude limit beyond which our metallicity calibration should not be used.

Finally, Figure 14 shows a metallicity map of the Milky Way derived using $\simeq 9$ million stars with Gaia parallaxes, good SkyMapper and 2MASS $v g K_{S}$ photometry and applying Eq. 12 within its range of validity. For the sake of this plot we do not apply any requirement on the quality of parallaxes, since the goal is mostly illustrative. We verified though, that restricting to parallaxes better than 10 percent and adopting the quality cuts in Arenou et al. (2018) we still see the same metallicity trends, although with a much reduced number of stars, and probing a smaller volume. The empty regions close to the plane are areas currently not targeted by SkyMapper. Nevertheless, we can clearly see high metallicity stars being preferentially restricted to the Galactic plane, and the mean metallicity decreasing when moving to higher Galactic height $|Z|$, transitioning from the thin to the thick disc into the halo, just as expected from our knowledge of the Galaxy. While a proper study of the metallicity structure would require accounting for target selection effects, and we defer this to a future investigation, Figure 14 gives an example of the kind of studies SkyMapper photometry will enable.

Figure 14 is reminescent of the Milky Way metallicity tomography done by Ivezić et al. (2008), using 2.5 million stars with SDSS colours and photometric distances. The rms scatter of our metallicity residual $(0.21 \mathrm{dex})$ is also similar to their $(0.24 \mathrm{dex})$, which is not entirely surprising since both works crucially rely on the use of one ultraviolet filter: SkyMapper $v$ for us (centred at $\sim 3800 \AA$ with a bandwidth of 320 $\AA$ ) versus Sloan $u$ for them (centred at $\sim 3600 \AA$ with a bandwidth of $\sim 540 \AA$ ). However, the functional form we use for our metallicity calibration has about half the colour terms compared to Ivezić et al. (2008, their eq 4). The theoretical sensitivity of some SkyMapper and Sloan filters to metallicity is quantified in Figure 15 which shows the change of synthetic magnitudes (upper panels) and colours (lower panels) for 

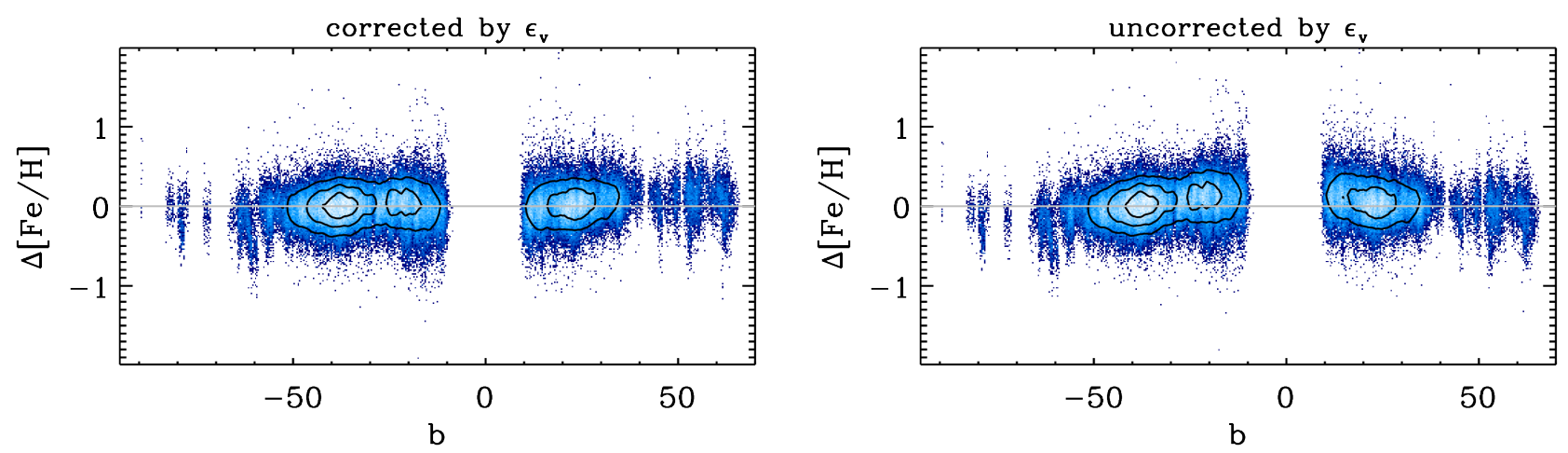

Figure 12. Left-panel: metallicity residuals (spectroscopic minus photometric) as function of Galactic latitude when the calibration is derived using $v^{\prime}$. Rightpanel: same as left panel, but using instead $v$ to derive the metallicity calibration. Colours indicate the density of stars, from highest (light-blue) to lowest (dark-blue). Contour levels are also shown to make the trend more clear.
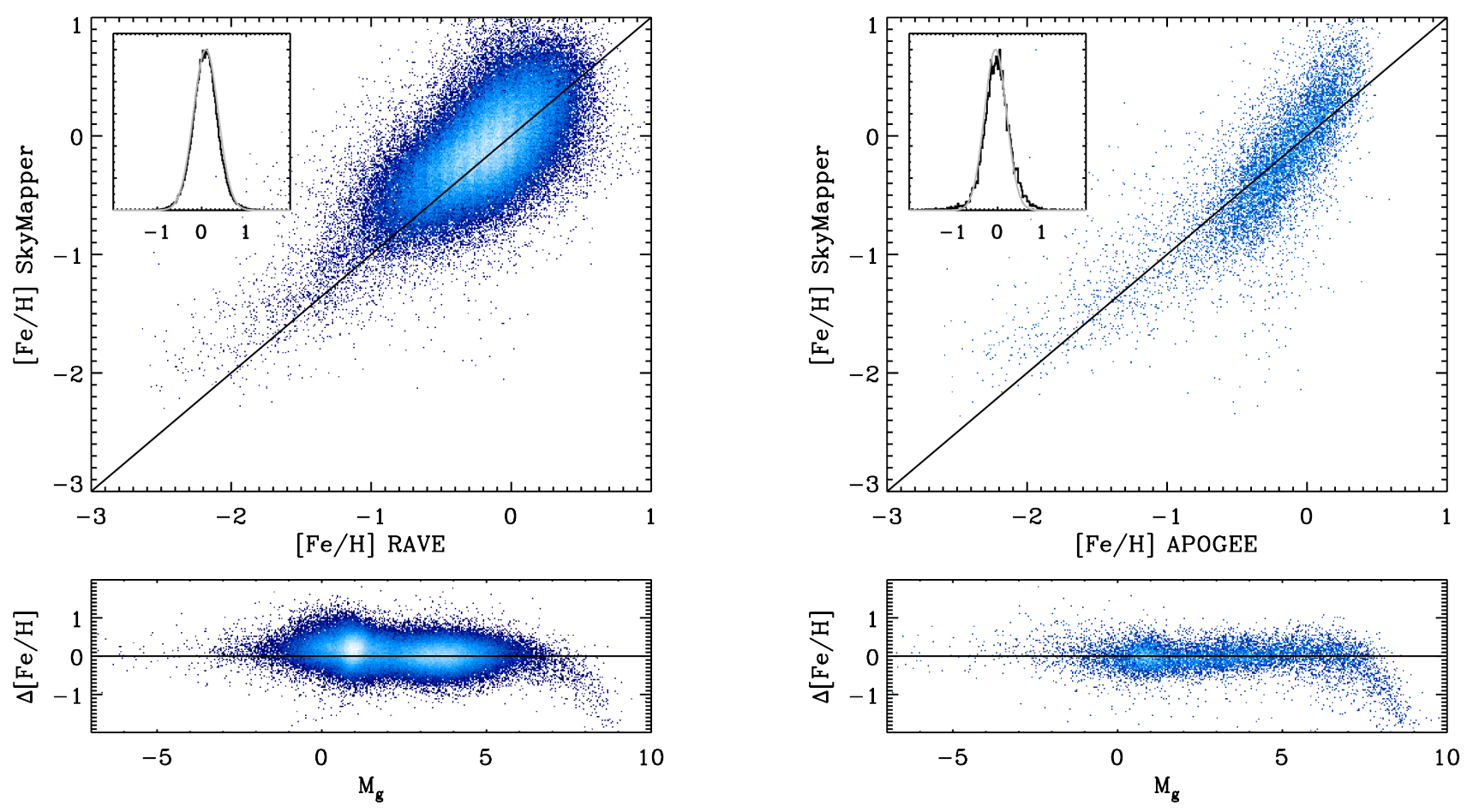

Figure 13. Left-panel: RAVE DR5 versus SkyMapper metallicities for over 140, 000 stars using our calibration (top), and residuals fitted with a Gaussian of width 0.28 dex (inset). Only stars with $M_{g}<7$, RAVE flags ALGO_CONV=0, c1=c2=c3=n and $P_{1}<\left(v^{\prime}-g\right)_{0}<P_{2}$ are used for this comparison. Residuals as function of $M_{g}$ (bottom) are also shown, to highlight how the metallicity calibration degrade for $M_{g}>7$. Right-panel: same as left-panel, but using over 9,500 stars having ASCAP parameters from APOGEE DR14. Only objects without bad flags are used. Residuals are fitted with a Gaussian of width 0.25 dex. Colours indicate the density of stars.

a 0.1 dex decrease in metallicity at a given $T_{\text {eff }}, \log g$ and $[\mathrm{Fe} / \mathrm{H}]$. The sequence of $T_{\text {eff }}$ and $\log g$ sampled (left panel) is typical of the parameter space covered by late-type stars. Keeping in mind the performances of theoretical colours in matching real data, our goal here is to single out the effect on photometry of changing metallicity at given $T_{\text {eff }}$ and $\log g$. Thus we favour this approach over the use of isochrones, where a change of metallicity would move isochrones in the $T_{\text {eff }}-\log g$ plane as well. Figure 15 indicates that the use of the SkyMapper $v$ band yields a metallicity sensitivity similar to the Sloan $u$, at least over the metallicity range covered by our calibration. However, the larger bandwidth of Sloan $u$ makes it sensitive to $\log g$, whereas this is not the case for SkyMapper $v$ within the limits we previously discussed.

\section{CONCLUSIONS}

In this paper we have conducted a thorough study of SkyMapper DR1.1 photometry. First, we have checked its standardization; ideally to do so a large number of absolute flux standards would be needed. Given their current absence, we have devised a new method based on the effective temperature of a sample of reference stars to determine photometric zero-points across the sky. This approach is 


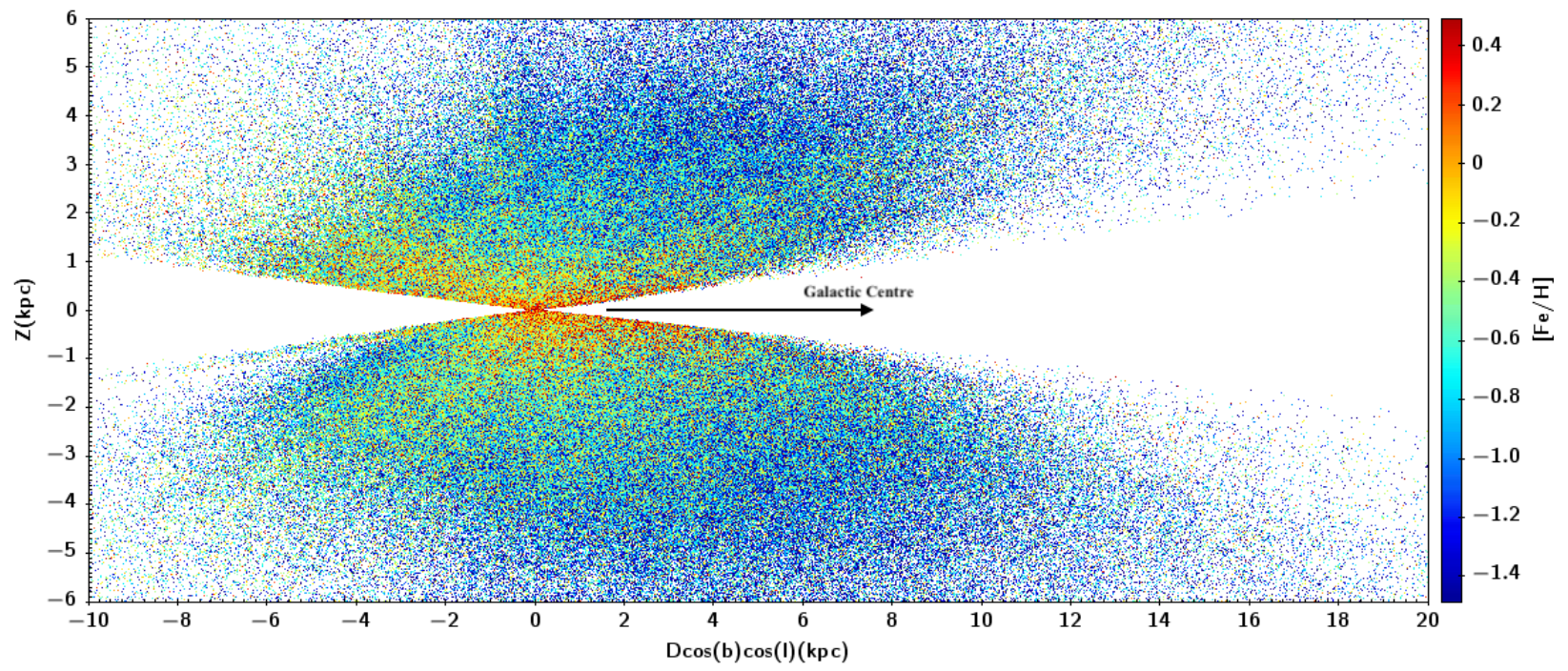

Figure 14. Milky Way metallicity map using $\simeq 9$ millions stars for which our calibration can be applied, and with Gaia DR2 parallaxes. In this Cartesian frame, the Sun is located at $(0,0)$, where $Z$ is height from the plane, $D$ is the distance and $(l, b)$ are Galactic coordinates. The direction to the Galactic Centre (approximately at $8 \mathrm{kpc}$ ) is also indicated.

applicable to any photometric survey, but it is particularly relevant for SkyMapper, since its zero-points are not tied to spectrophotometric standard stars, but are obtained from predicted SkyMapper magnitudes of an ensemble of stars with photometry from other surveys. The approach currently adopted by SkyMapper works remarkably well for griz, but has limitations in the $u v$ bands. With our method we have recovered an offset of the $u v$ zero-points that varies as a function of Galactic latitude. This variation is expected as a result of the reddening corrections currently employed in predicting SkyMapper $u v$ magnitudes from external photometry at longer wavelengths.

With a good control over photometric zero-points, we have then applied the InfraRed Flux Method to derive effective temperatures for all stars in the GALAH spectroscopic survey, and provide empirical colour $-T_{\text {eff }}$ relations. We have also used the GALAH spectroscopic metallicities to derive a relation between them and SkyMapper $v, g$, and 2MASS $K_{S}$ magnitudes. Our calibrations is validated down to approximately $[\mathrm{Fe} / \mathrm{H}]=-2$, and applies to latetype giants, and dwarfs with $M_{g}<7$. The reliability of our photometric metallicities is further checked against RAVE DR5 and APOGEE DR14, confirming an overall precision of 0.2 dex. Finally, using $\sim 9$ million stars with Gaia parallaxes, we have produced a metallicity map in which we can clearly trace the mean metallicity decreasing as we move from the thin disc to the thick disc and then on into the halo, in agreement with what is expected from our knowledge of the Milky Way's structure.

\section{ACKNOWLEDGEMENTS}

We thank the referee for constructive comments that strengthen the presentation. LC, ADM and DY acknowledge support from the Australian Research Council (grants FT160100402, FT160100206 and FT140100554). Parts of this research were conducted by the Australian Research Council Centre of Excellence for All Sky Astrophysics in 3 Dimensions (ASTRO 3D), through project number CE170100013. This research made use of TOPCAP (Taylor 2005). The national facility capability for SkyMapper has been funded through ARC LIEF grant LE130100104 from the Australian Research Council, awarded to the University of Sydney, the Australian National University, Swinburne University of Technology, the University of Queensland, the University of Western Australia, the University of Melbourne, Curtin University of Technology, Monash University and the Australian Astronomical Observatory. SkyMapper is owned and operated by The Australian National University's Research School of Astronomy and Astrophysics. The survey data were processed and provided by the SkyMapper Team at ANU. The SkyMapper node of the All-Sky Virtual Observatory (ASVO) is hosted at the National Computational Infrastructure (NCI). Development and support the SkyMapper node of the ASVO has been funded in part by Astronomy Australia Limited (AAL) and the Australian Government through the Commonwealth's Education Investment Fund (EIF) and National Collaborative Research Infrastructure Strategy (NCRIS), particularly the National eResearch Collaboration Tools and Resources (NeCTAR) and the Australian National Data Service Projects (ANDS). This work has made use of data from the European Space Agency (ESA) mission Gaia (https://www . cosmos. esa. int/gaia), processed by the Gaia Data Processing and Analysis Consortium (DPAC, https://www. cosmos.esa.int/web/ gaia/dpac/consortium). Funding for the DPAC has been provided by national institutions, in particular the institutions participating in the Gaia Multilateral Agreement. This publication makes use of data products from the Two Micron All Sky Survey, which is a joint project of the University of Massachusetts and the Infrared Processing and Analysis Center/California Institute of Technology, funded by the National Aeronautics and Space Administration and the National Science Foundation. Funding for RAVE (WWW . rave-survey.org) has been provided by institutions of the RAVE participants and by their national funding agencies. Funding for the Sloan Digital Sky Survey IV has been provided by the Alfred P. Sloan Foundation, the U.S. Department of Energy Office of Science, and the Participating Institutions. SDSS-IV acknowledges support and resources from the Center for High-Performance Computing at the University of Utah. The SDSS web site is WWW. sdss. org SDSS-IV is managed by the Astrophysical Research Consor- 

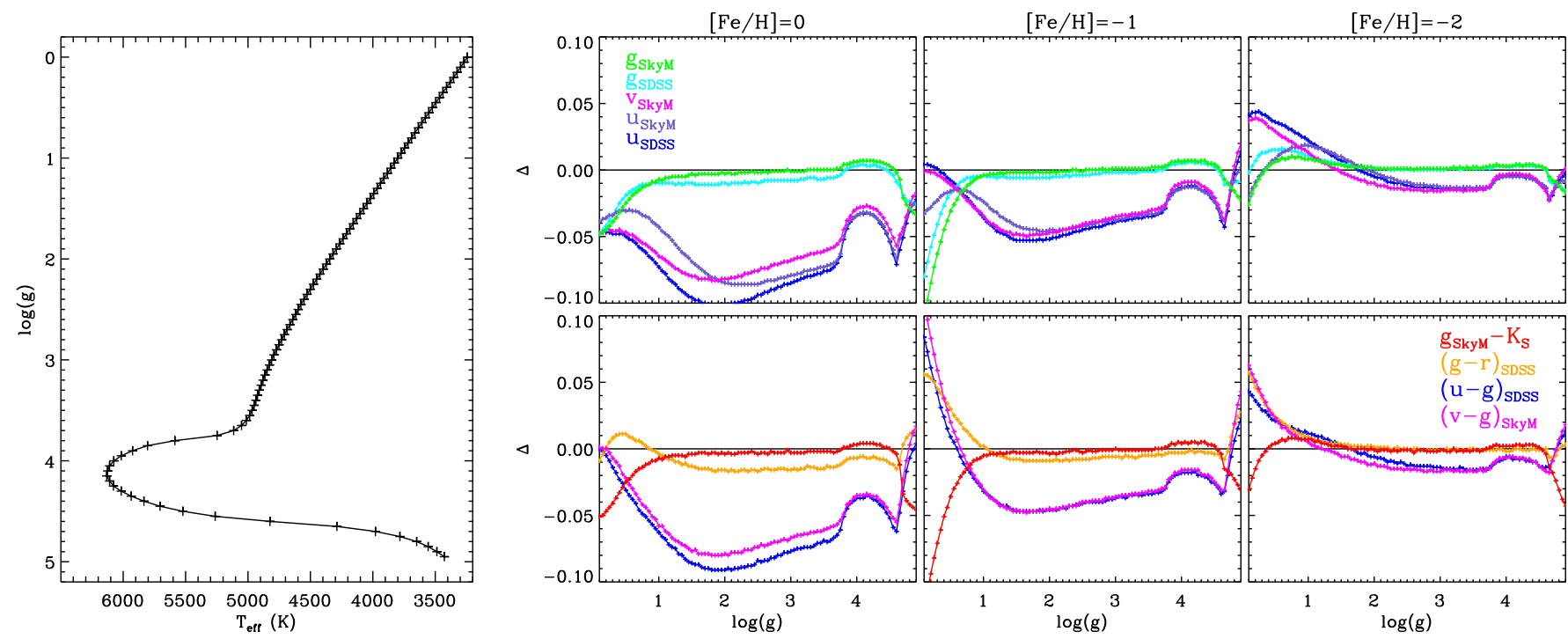

Figure 15. Predicted sensitivity to metallicity in a number of filters and colour indices using synthetic photometry from Casagrande \& VandenBerg 2014 $2018 \mathrm{~b}$ Left-panel: crosses mark the location in the $T_{\text {eff }}-\log g$ plane where the photometric sensitivity to a change in metallicity is quantified. Upper-right panels: change in magnitude $(\Delta)$ for Sloan $u, g$ and SkyMapper $u, v$ and $g$ filters when metallicity at $0,-1$ and -2 (as indicated) is decreased by 0.1 dex. Lower-right panels: same as above, but for the colour indices used in our (red and magenta) and Ivezic's metallicity calibration (orange and blue).

tium for the Participating Institutions of the SDSS Collaboration including the Brazilian Participation Group, the Carnegie Institution for Science, Carnegie Mellon University, the Chilean Participation Group, the French Participation Group, Harvard-Smithsonian Center for Astrophysics, Instituto de Astrofísica de Canarias, The Johns Hopkins University, Kavli Institute for the Physics and Mathematics of the Universe (IPMU) / University of Tokyo, the Korean Participation Group, Lawrence Berkeley National Laboratory, Leibniz Institut für Astrophysik Potsdam (AIP), Max-PlanckInstitut für Astronomie (MPIA Heidelberg), Max-Planck-Institut für Astrophysik (MPA Garching), Max-Planck-Institut für Extraterrestrische Physik (MPE), National Astronomical Observatories of China, New Mexico State University, New York University, University of Notre Dame, Observatário Nacional / MCTI, The Ohio State University, Pennsylvania State University, Shanghai Astronomical Observatory, United Kingdom Participation Group, Universidad Nacional Autónoma de México, University of Arizona, University of Colorado Boulder, University of Oxford, University of Portsmouth, University of Utah, University of Virginia, University of Washington, University of Wisconsin, Vanderbilt University, and Yale University.

\section{APPENDIX A: MONOCHROMATIC AND IN-BAND \\ FLUXES}

With the photon-counting formalist adopted in this paper, the monochromatic flux associated to the effective wavelength of each SkyMapper magnitude can be determined from Eq. (1)-2):

$$
\frac{\int_{\lambda_{i}}^{\lambda_{f}} f_{\lambda} \lambda T_{\zeta} \mathrm{d} \lambda}{\int_{\lambda_{i}}^{\lambda_{f}} \lambda T_{\zeta} \mathrm{d} \lambda}=10^{-0.4\left(m_{\zeta, \mathrm{SM}}-\epsilon_{\zeta}\right)} c f_{v}^{0} G(\lambda)
$$

where

$$
G(\lambda)=\frac{\int_{\lambda_{i}}^{\lambda_{f}} \frac{T_{\zeta}}{\lambda} \mathrm{d} \lambda}{\int_{\lambda_{i}}^{\lambda_{f}} \lambda T_{\zeta} \mathrm{d} \lambda} .
$$

Similarly, the in-band flux is:

$$
\int_{\lambda_{i}}^{\lambda_{f}} f_{\lambda} \lambda T_{\zeta} \mathrm{d} \lambda=10^{-0.4\left(m_{\zeta, \mathrm{SM}}-\epsilon_{\zeta}\right)} c f_{v}^{0} H(\lambda)
$$

where

$$
H(\lambda)=B w(\lambda) G(\lambda)=\int_{\lambda_{i}}^{\lambda_{f}} \frac{T_{\zeta}}{\lambda} \mathrm{d} \lambda,
$$

and $B w(\lambda)$ is the bandwidth. Monochromatic fluxes are associated with isophotal wavelengths, whose calculation is non-trivial because of discontinuities in stellar spectra (e.g., Tokunaga \& Vacca 2005. Casagrande et al. 2006, Rieke et al. 2008). The effective wavelength $\lambda_{\text {eff }}$ is thus a useful approximation (e.g., Golay 1974):

$$
\lambda_{\text {eff }}=\frac{\int_{\lambda_{i}}^{\lambda_{f}} \lambda^{2} f_{\lambda} T_{\zeta} \mathrm{d} \lambda}{\int_{\lambda_{i}}^{\lambda_{f}} \lambda f_{\lambda} T_{\zeta} \mathrm{d} \lambda}
$$

Values for $\epsilon_{\zeta}, G(\lambda), H(\lambda), B w(\lambda)$ and $\lambda_{\text {eff }}$ are listed in Table 1 where the CALSPEC spectrum of Vega has been adopted to compute the effective wavelength. For example, an object with $m_{g, \mathrm{SM}}=g=15$ will have a monochromatic flux of $4.261 \times 10^{-15} \mathrm{erg} \mathrm{s}^{-1} \mathrm{~cm}^{-2} \AA^{-1}$ and an in-band flux of $6.182 \times 10^{-12} \mathrm{erg} \mathrm{s}^{-1} \mathrm{~cm}^{-2}$.

\section{REFERENCES}

Abolfathi, B., et al. 2018, ApJS, 235, 42

Alonso, A., Arribas, S., \& Martinez-Roger, C. 1996, A\&AS, 117, 227

Arenou, F., et al. 2018, ArXiv e-prints

Árnadóttir, A. S., Feltzing, S., \& Lundström, I. 2010, A\&A, 521, A40 
Bessell, M., Bloxham, G., Schmidt, B., Keller, S., Tisserand, P., \& Francis, P. 2011, PASP, 123, 789

Bessell, M., \& Murphy, S. 2012, PASP, 124, 140

Bessell, M. S. 2005, ARA\&A, 43, 293

Blackwell, D. E., Lynas-Gray, A. E., \& Petford, A. D. 1991, A\&A, 245, 567

Blackwell, D. E., Petford, A. D., Arribas, S., Haddock, D. J., \& Selby, M. J. 1990, A\&A, 232, 396

Blackwell, D. E., Petford, A. D., \& Shallis, M. J. 1980, A\&A, 82, 249

Blackwell, D. E., Shallis, M. J., \& Selby, M. J. 1979, MNRAS, 188,847

Boeche, C., et al. 2014, A\&A, 568, A71

Bohlin, R. C. 2007, in Astronomical Society of the Pacific Conference Series, Vol. 364, The Future of Photometric, Spectrophotometric and Polarimetric Standardization, ed. C. Sterken, 315

Bohlin, R. C. 2014, AJ, 147, 127

Bohlin, R. C., Dickinson, M. E., \& Calzetti, D. 2001, AJ, 122, 2118

Buder, S., et al. 2018, MNRAS, 478, 4513

Cardelli, J. A., Clayton, G. C., \& Mathis, J. S. 1989, ApJ, 345, 245

Casagrande, L., Portinari, L., \& Flynn, C. 2006, MNRAS, 373, 13

Casagrande, L., et al. 2014a, MNRAS, 439, 2060

Casagrande, L., Ramírez, I., Meléndez, J., Bessell, M., \& Asplund, M. 2010, A\&A, 512, A54

Casagrande, L., Schönrich, R., Asplund, M., Cassisi, S., Ramírez, I., Meléndez, J., Bensby, T., \& Feltzing, S. 2011, A\&A, 530, A138

Casagrande, L., et al. 2016, MNRAS, 455, 987

Casagrande, L., et al. 2014b, ApJ, 787, 110

Casagrande, L., \& VandenBerg, D. A. 2014, MNRAS, 444, 392

Casagrande, L., \& VandenBerg, D. A. 2018a, MNRAS, 479, L102

Casagrande, L., \& VandenBerg, D. A. 2018b, MNRAS, 475, 5023

Ciucă, I., Kawata, D., Lin, J., Casagrande, L., Seabroke, G., \&

Cropper, M. 2018, MNRAS, 475, 1203

Covey, K. R., et al. 2007, AJ, 134, 2398

Da Costa, G., et al. 2018, in preparation

De Silva, G. M., et al. 2015, MNRAS, 449, 2604

Doi, M., et al. 2010, AJ, 139, 1628

Eisenstein, D. J., et al. 2006, ApJS, 167, 40

Fitzpatrick, E. L. 1999, PASP, 111, 63

Francis, P. J., \& Wills, B. J. 1999, in Astronomical Society of the Pacific Conference Series, Vol. 162, Quasars and Cosmology, ed. G. Ferland \& J. Baldwin, 363

Fukugita, M., Ichikawa, T., Gunn, J. E., Doi, M., Shimasaku, K., \& Schneider, D. P. 1996, AJ, 111, 1748

Gaia Collaboration, Brown, A. G. A., Vallenari, A., Prusti, T., de Bruijne, J. H. J., Babusiaux, C., \& Bailer-Jones, C. A. L. 2018, ArXiv e-prints

Golay, M., ed. 1974, Astrophysics and Space Science Library, Vol. 41, Introduction to astronomical photometry

Henden, A. A., Templeton, M., Terrell, D., Smith, T. C., Levine, S., \& Welch, D. 2016, VizieR Online Data Catalog, 2336

High, F. W., Stubbs, C. W., Rest, A., Stalder, B., \& Challis, P. 2009, AJ, 138, 110

Holberg, J. B., \& Bergeron, P. 2006, AJ, 132, 1221

Howes, L. M., et al. 2016, MNRAS, 460, 884

Ivezić, Ž., Beers, T. C., \& Jurić, M. 2012, ARA\&A, 50, 251

Ivezić, Ž., et al. 2008, ApJ, 684, 287

Ivezić, Ž., et al. 2007, AJ, 134, 973

Karovicova, I., et al. 2018, MNRAS, 475, L81
Keller, S. C., et al. 2014, Nature, 506, 463

Keller, S. C., et al. 2007, PASA, 24, 1

Kunder, A., et al. 2017, AJ, 153, 75

Lallement, R., Welsh, B. Y., Vergely, J. L., Crifo, F., \& Sfeir, D. 2003, A\&A, 411, 447

MacDonald, E. C., et al. 2004, MNRAS, 352, 1255

McClure, R. D. 1976, AJ, 81, 182

Ness, M., Hogg, D. W., Rix, H.-W., Ho, A. Y. Q., \& Zasowski, G. 2015, ApJ, 808, 16

Nordström, B., et al. 2004, A\&A, 418, 989

O’Donnell, J. E. 1994, ApJ, 422, 158

Rieke, G. H., et al. 2008, AJ, 135, 2245

Schlafly, E. F., et al. 2016, ApJ, 821, 78

Schlegel, D. J., Finkbeiner, D. P., \& Davis, M. 1998, ApJ, 500, 525

Skrutskie, M. F., et al. 2006, AJ, 131, 1163

Stetson, P. B. 2005, PASP, 117, 563

Strömgren, B. 1951, AJ, 56, 142

Taylor, M. B. 2005, in Astronomical Society of the Pacific Conference Series, Vol. 347, Astronomical Data Analysis Software and Systems XIV, ed. P. Shopbell, M. Britton, \& R. Ebert, 29

Tokunaga, A. T., \& Vacca, W. D. 2005, PASP, 117, 421

White, T. R., et al. 2018, MNRAS, 477, 4403

Wolf, C., et al. 2018, PASA, 35, e010

Yong, D., et al. 2013, ApJ, 762, 27

Yuan, H., Liu, X., Xiang, M., Huang, Y., Zhang, H., \& Chen, B. 2015, ApJ, 799, 133 